\title{
Systems of stochastic Poisson equations: hitting probabilities
}

by

\author{
MARTA SANZ-SOLÉ $(a)(*)$ \\ Facultat de Matemàtiques i Informàtica \\ Universitat de Barcelona \\ Gran Via de les Corts Catalanes, 585 \\ 08007 Barcelona, Spain \\ e-mail: marta.sanz@ub.edu \\ http://www.ub.edu/plie/Sanz-Sole
}

\author{
and NoÈlia Viles $\left.{ }^{(}\right)$ \\ @DiMedia, Actividades Digital Media \\ Avinguda Diagonal, 477 \\ 08036 Barcelona, Spain \\ e-mail:noelia.viles@gmail.com
}

\begin{abstract}
We consider a $d$-dimensional random field $u=(u(x), x \in D)$ that solves a system of elliptic stochastic equations on a bounded domain $D \subset \mathbb{R}^{k}$, with additive white noise and spatial dimension $k=1,2,3$. Properties of $u$ and its probability law are proved. For Gaussian solutions, using results from [9], we establish upper and lower bounds on hitting probabilities in terms of the Hausdorff measure and Bessel-Riesz capacity, respectively. This relies on precise estimates on the canonical distance of the process or, equivalently, on $L^{2}$ estimates of increments of the Green function of the Laplace equation.
\end{abstract}

Keywords: Systems of stochastic Poisson equations; hitting probabilities, capacity; Hausdorff measure.

AMS Subject Classification. Primary: 60H15, 60G15, 60J45; Secondary: 60G60, $60 \mathrm{H} 07$.

(a) Corresponding author.

(*) Supported by the grants MTM 2012-31192, MTM 2015- 65092-P, from the Dirección General de Investigación, Ministerio de Educación y Ciencia, Spain.

(*) Supported by the grant MTM 2012-31192 from the Dirección General de Investigación, Ministerio de Economía y Competitividad, Spain. This work was partially done during a postdoctoral period at the University of Barcelona, supported by the Juan de la Cierva contract JCI-2011- 08840. 


\section{Introduction}

Let $D$ be a bounded domain of $\mathbb{R}^{k}, k=1,2,3$, for which the divergence theorem holds. Consider the following system of elliptic stochastic partial differential equations,

$$
\begin{aligned}
& -\Delta u^{i}(x)+f^{i}(u(x))=g^{i}(x)+\sum_{j=1}^{d} \sigma_{i j} \dot{W}^{j}(x), x \in D, i=1, \ldots, d, \\
& u_{\mid \partial D}(x)=0,
\end{aligned}
$$

where $\dot{W}=\left(\dot{W}^{j}, j=1, \ldots, d\right)$ denotes a $d$-dimensional white noise indexed by $x \in \mathbb{R}^{k}, f: \mathbb{R}^{d} \rightarrow \mathbb{R}^{d}, g: D \rightarrow \mathbb{R}^{d}$, and $\sigma=\left(\sigma_{i j}\right)_{1 \leq i, j \leq d}$ is a non-singular matrix with real-valued entries.

The main motivation of this paper has been to find upper and lower bounds for the hitting probabilities $\mathbb{P}\{u(I) \cap A \neq \emptyset\}, I \subset D, A \subset \mathbb{R}^{d}$, in terms of the Hausdorff measure and the capacity of the set $A$, respectively. This is a fundamental problem in probabilistic potential theory that, in the context of stochastic partial differential equations, has been extensively studied for the stochastic heat and wave equations. We refer the reader to [4], [7], [10], and references herein, for a representative sample of results.

For $d=1$, equations like (10 have been first considered in [3] and then in [11], in relation with the study of the Markov field property of the solution, and in [12], [19], [21], for numerical approximations, among others. We observe that in (1), the stochastic forcing is an additive noise. Therefore, in the integral formulation of the system given in (6), the stochastic integral term contains a deterministic integrand and defines a Gaussian process. Since there is no time parameter in (1), considering a multiplicative noise would require a choice of anticipating stochastic integral in (6). For example, one could take the Skorohod integral. This would make the objective of this article difficult and rather speculative.

The content of the paper is as follows. In Section 2, we prove the existence and uniqueness of a solution to (1), when the function $f$ satisfies a monotonicity condition (see Theorem 2.2). This is a $d$-dimensional stochastic process indexed by $\bar{D}$, the closure of the domain $D$, with continuous sample paths and vanishing at the boundary of $D$, a.s. The proof applies standard methods of the theory of nonlinear monotone operators. In order to make the article self-contained, we include the details of the proof. In Section 3 , we prove some properties of the solution to (1). With the a priori bound proved in Proposition 3.1 we prove that the solution lies in $L^{p}\left(\Omega ; \mathbb{R}^{d}\right)$, uniformly in $x \in D$. Moreover, by using estimates of increments of the $L^{2}$-norms of the Green function, we prove that the sample paths of the solution are Hölder continuous. Section 3 is devoted to study some aspects of the law of the solution. The integral formulation (6) suggests that the law of $u$ is obtained from a Gaussian process by a non adapted shift. By applying Kusuoka's anticipating extension of Girsanov's theorem (see [15]) we obtain the absolute continuity of the law of $u$ with respect to a Gaussian measure. As a trivial by-product, for any 
$x \in D$, the law of $u(x)$ is absolutely continuous with respect to the Lebesgue measure in $\mathbb{R}^{d}$. For $d=1$, this result has been proved in [11].

In the remaining of the article, it is assumed that $f \equiv 0$, therefore focusing on Gaussian solutions. For the sake of simplicity, we also assume $g \equiv 0$. Probabilistic potential theory for Gaussian processes has been the object of extensive work. The more recent developments are on anisotropic random fields with the paradigmatic example of fractional Brownian sheets. Solutions to SPDEs, like the stochastic heat equation, belong to this class. In [22], different results relative to sample paths of anisotropic Gaussian random fields are presented, in particular on hitting probabilities, and a exhaustive number of references are given. The Gaussian process obtained from (6) with $f=g=0$, provides yet another example of random field for which, to the best of our acknowledge, results on hitting probabilities have not been obtained before.

Criteria for hitting probabilities of general random fields have been established in [9]. When implemented on Gaussian processes, two fundamental ingredients are needed. The first one concerns the canonical distance $\delta$ on values of the Gaussian process at two different points $x, z$, which is required to commensurate with a pseudo-distance on the parameter set of the process (see (40) for the definition of $\delta$ ). The second ingredient is the property of two-point local non determinism (see conditions (C1), (C2) in [1, Section 2], [22, p. 158]). For random fields obtained from solutions to SPDEs, both conditions are intimately connected with upper and lower bounds of increments of $L^{2}$-norms of the Green function or the fundamental solution (see [6], [9, Secction 4] for the stochastic heat and wave equations, respectively). For the Green function of the Laplace's equation, Section 5.3 provides the necessary results. We prove that in spatial dimension $k=1,3$, those norms of increments commesurates with $|x-z|$ and $|x-z|^{1 / 2}$, respectively, while for $k=2$ there is a gap (see Lemmas 5.4, 5.7, 5.5, respectively). By applying these results, we establish in Section 5.4 upper and lower bounds for the hitting probabilities of the Gaussian process defined in (23) in dimension $k=1,3$, and upper bounds in dimension $k=2$. Our investigations led to the conclusion that the gap in the estimates of Lemma 5.5 implies that for $k=2$ the two-point local non determinism fails to be true, although we do not have a proof of this statement.

We end this section with some remarks on a possible extension of our results to $f \neq 0$. A natural approach, inspired by [6], consists in applying Proposition 4.2. By doing so, from hitting probabilities estimates on the Gaussian solution, and moment estimates of the random density (21), upper and lower bounds on hitting probabilities for $u$ could be obtained. Assume that $f(x)=a x+b$. Then, the $\operatorname{det}_{2}$ factor on the right-hand side of (21) is constant, and the exponential factor involves the random variable $\delta(f(\omega))$ that belongs to a second order Wiener chaos. With a constraint on the size of the constant $a$, moments up to a certain order of the random density do exist, and the above strategy works well. However, we believe that going beyond that particular case would need new ideas. 


\section{Existence and uniqueness of solution to the system of elliptic equations}

This section is devoted to establish the existence and uniqueness of solution to the system of elliptic equations (11).

We begin with recalling the expression of the Green function associated to the Laplace equation in dimensions $k=1,2,3$, that we denote by $G_{D}^{k}$.

For $k=1$, we consider $D=(0, b)$, with $b>0$. In this case,

$$
G_{D}^{1}(x, y)=x \wedge y-\frac{x y}{b} .
$$

For $k=2,3, D$ is an arbitrary domain with regular boundary, and then,

$$
G_{D}^{k}(x, y)=\Gamma^{k}(|x-y|)-\mathbb{E}_{x}\left(\Gamma^{k}\left(\left|B_{\tau}-y\right|\right), x, y \in D .\right.
$$

In this formula, $B_{\tau}$ is the random variable defined by a Brownian motion $B$ that starts from $x$ at time $t=0$, at the first time (denoted by $\tau$ ) it hits $\partial D$, and

$$
\Gamma^{k}(z)=C_{k}\left\{\begin{array}{l}
\log |z|, k=2 \\
|z|^{-1}, k=3
\end{array}\right.
$$

with $C_{2}=\frac{1}{2 \pi}$ and $C_{3}=\frac{1}{4 \pi}$ (see [13]). In dimensions $k=2,3$, and for $D=$ $B_{1}(0)$, the unit ball centered at zero, we give in Section 5.3 an alternate formula for $G_{D}^{k}(x, y)$ (see (44).

The inner product in $L^{2}\left(D ; \mathbb{R}^{d}\right)$ and its corresponding norm will be denoted by $\langle\cdot, \cdot\rangle$ and $\|\cdot\|$, respectively.

For its further and frequent use, we quote a well-known result of the $L^{2}$-norm of $G_{D}^{k}$.

Lemma 2.1 For $k=1$, we consider $D=(0, b), b>0$, and for $k=2,3, D$ is an arbitrary bounded domain of $\mathbb{R}^{k}$ with regular boundary. We have

$$
\sup _{x \in D}\left\|G_{D}^{k}(x, \cdot)\right\|<+\infty .
$$

Proof: Let $k=1$. Explicit computations based on the expression (2) yield

$$
\int_{0}^{b} G_{D}^{1}(x, y)^{2} d y=\frac{x^{2}(x-b)^{2}}{3 b} .
$$

From this, one trivially gets (4).

For $k=2,3$, the result is proved in [11, Lemma 3.3].

Following [3] (see also [11], [12], [21]), a stochastic process $u=\{u(x), x \in$ $D$ \} satisfying

$$
\begin{aligned}
u^{i}(x)+\int_{D} G_{D}^{k}(x, y) f^{i}(u(y)) d y & =\int_{D} G_{D}^{k}(x, y) g^{i}(y) d y \\
& +\sum_{j=1}^{d} \sigma_{i j} \int_{D} G_{D}^{k}(x, y) W^{j}(d y), i=1, \ldots, d
\end{aligned}
$$


a.s. for all $x \in D$, is called a mild solution of (1).

We define the (Nemytskii type) operator $\mathrm{F}: L^{2}\left(D ; \mathbb{R}^{d}\right) \longrightarrow L^{2}\left(D ; \mathbb{R}^{d}\right)$ by $F(v)(y)=f(v(y))$, for any $v \in L^{2}\left(D ; \mathbb{R}^{d}\right), y \in \mathbb{R}^{d}$, and introduce the following assumptions.

(C) $\mathrm{F}$ is strongly continuous and bounded.

(M) Monotonicity. There exists $L>0$ such that for any $u, v \in L^{2}\left(D ; \mathbb{R}^{d}\right)$,

$$
\langle u-v, \mathrm{~F}(u)-\mathrm{F}(v)\rangle \geq-L\|u-v\|^{2} .
$$

For its further use, we recall a consequence of Poincaré's inequality:

(P) There exists a constant $a>0$ such that for any $\varphi \in L^{2}\left(D ; \mathbb{R}^{d}\right)$,

$$
\left\langle\int_{D} G_{D}^{k}(\cdot, y) \varphi(y) d y, \varphi\right\rangle \geq a\left\|\int_{D} G_{D}^{k}(\cdot, y) \varphi(y) d y\right\|^{2} .
$$

In the sequel, we denote by $\mathcal{C}\left(\bar{D} ; \mathbb{R}^{d}\right)$ the space of continuous functions on $\bar{D}$ and set $\mathcal{S}=\left\{\omega: \omega \in \mathcal{C}\left(\bar{D} ; \mathbb{R}^{d}\right),\left.\omega\right|_{\partial D}=0\right\}$. The result on existence and uniqueness of solution for (6) reads as follows.

\section{Theorem 2.2 Assume that}

(i) $g \in L^{2}\left(D ; \mathbb{R}^{d}\right)$;

(ii) F satisfies the properties $(\boldsymbol{C})$ and $(\boldsymbol{M})$;

(iii) the constants $L$ and $a$ in $(\boldsymbol{M})$ and $(\boldsymbol{P})$ respectively, satisfy $0<L<a$.

Then, the system of equations (6) has a unique solution $\{u(x), x \in D\}$ in $\mathcal{S}$.

Proof: For any $\omega \in \mathcal{S}$, we set

$$
\mathrm{T}(\omega):=\omega+\int_{D} G_{D}^{k}(\cdot, y) f(\omega(y)) d y .
$$

This defines an operator $\mathrm{T}: \mathcal{S} \longrightarrow \mathcal{S}$. Indeed, the function $x \longrightarrow \int_{D} G_{D}^{k}(x, y) f(\omega(y)) d y$ vanishes if $x \in \partial D$. Fix $x, \bar{x} \in D$. Cauchy-Schwarz inequality yields

$$
\begin{aligned}
\left|\int_{D}\left[G_{D}^{k}(x, y)-G_{D}^{k}(\bar{x}, y)\right] f(\omega(y)) d y\right| & \leq\left\|G_{D}^{k}(x, \cdot)-G_{D}^{k}(\bar{x}, \cdot)\right\|\|f(\omega)\| \\
& \leq C\left\|G_{D}^{k}(x, \cdot)-G_{D}^{k}(\bar{x}, \cdot)\right\| .
\end{aligned}
$$

Appealing to lemmas 5.4, 5.5, 5.7 we see that $x \longrightarrow \int_{D} G_{D}^{k}(\cdot, y) f(\omega(y)) d y$ belongs to $\mathcal{C}\left(\bar{D} ; \mathbb{R}^{d}\right)$ and in fact, it is Hölder continuous.

Let

$$
b^{i}(x)=\int_{D} G_{D}^{k}(x, y) g^{i}(y) d y+\sum_{j=1}^{d} \sigma_{i, j} \int_{D} G_{D}^{k}(x, y) W^{j}(d y), i=1, \ldots, d .
$$


Clearly, $b^{i}(x)=0$ if $x \in \partial D$. The process $\left\{\int_{D} G_{D}^{k}(x, y) W^{j}(d y), x \in D\right\}$ has continuous sample paths, a.s. Indeed this follows from lemmas 5.4, 5.5, 5.7, the hypercontractivity property and Kolmogorov's continuity criterion. The term $\int_{D} G_{D}^{k}(x, y) g^{i}(y) d y$ defines also a continuous function in $x$. This follows from Cauchy-Schwarz inequality and again, by applying lemmas 5.4, 5.5, 5.7. Consequently, $b(x)=\left(b^{i}(x), i=1, \ldots, d\right) \in \mathcal{S}$.

We will prove that the operator equation $\mathrm{T} \omega=b$ has a unique solution for any $b \in \mathcal{S}$, or equivalently that $\mathrm{T}$ is a bijective operator on $\mathcal{S}$.

For this, we start by checking that $\mathrm{T}$ is one-to-one. Fix $u, v \in \mathcal{S}$ and assume that $\mathrm{T} u=\mathrm{T} v$. Then

$$
u(x)-v(x)=-\int_{D} G_{D}^{k}(x, y)[f(u(y))-f(v(y))] d y .
$$

By taking the inner product in $L^{2}\left(D ; \mathbb{R}^{d}\right)$ with $F(u)-F(v)$ on both sides of this equality, and applying $(\mathbf{P})$, we obtain

$$
\begin{aligned}
\langle u-v, F(u)-F(v)\rangle & =-\left\langle\int_{D} G_{D}^{k}(\cdot, y)[f(u(y))-f(v(y))] d y, F(u)-F(v)\right\rangle \\
& \leq-a\left\|\int_{D} G_{D}^{k}(\cdot, y)[f(u(y))-f(v(y))] d y\right\|^{2} \\
& =-a\|u-v\|^{2} .
\end{aligned}
$$

On the other hand, using the property $(\mathbf{M})$, we have

$$
\langle u-v, F(u)-F(v)\rangle \geq-L\|u-v\|^{2} .
$$

Thus,

$$
-L\|u-v\|^{2} \leq-a\|u-v\|^{2},
$$

or equivalently,

$$
(a-L)\|u-v\|^{2} \leq 0 .
$$

Since $L<a$, this implies that $u=v$ in $\mathcal{S}$.

Next, we prove that $\mathrm{T}$ is onto, proceeding in a way similar as in [19].

Step 1: A solution for a regular problem. For a fixed $b \in \mathcal{S}$, we consider a sequence $\left(b_{n}\right)_{n \geq 1} \in \mathcal{C}_{0}^{\infty}\left(D ; \mathbb{R}^{d}\right)$, such that $b_{n} \rightarrow b$ in $L^{2}\left(D ; \mathbb{R}^{d}\right)$. Define $\mathrm{A}=-\Delta+F$ restricted to $W_{0}^{1,2}\left(D ; \mathbb{R}^{d}\right)$. We will prove that for each $n \geq 1$, there exists $u_{n} \in \mathcal{S}$ such that $\mathrm{A} u^{n}=b^{n}$.

We remind the classical result on solutions of nonlinear monotone operator equations (see, e.g. [23, Theorem 26.A, page 557]):

Let $X$ be a reflexive Banach space; denote by $X^{*}$ its topological dual. Let $B$ : $X \rightarrow X^{*}$ be a strictly monotone, coercive, hemicontinuous operator. Then, for any $k \in X^{*}$, the equation $B w=k$ has a unique solution on $X$. 
This theorem will be applied to $B:=A$ and $X:=W_{0}^{1,2}\left(D ; \mathbb{R}^{d}\right)$. Notice that $\mathrm{A}=-\Delta+F$ coincides with the operator $\mathrm{T}$ on the space $W_{0}^{1,2}\left(D ; \mathbb{R}^{d}\right) \cap \mathcal{S}$. Moreover, for any $u, v \in W_{0}^{1,2}(D)$,

$$
\langle\mathrm{A} u, v\rangle=\int_{D} \nabla u(x) \nabla v(x) d x+\int_{D} f(u(y)) v(y) d y,
$$

or coordinatewise,

$$
\left\langle\mathrm{A} u^{i}, v^{i}\right\rangle=\int_{D} \nabla u^{i}(x) \nabla v^{i}(x) d x+\int_{D} f^{i}(u(y)) v^{i}(y) d y .
$$

From Poincaré's inequality we deduce that for any $u \in W_{0}^{1,2}\left(D ; \mathbb{R}^{d}\right),\|\nabla u\|_{L^{2}\left(D ; \mathbb{R}^{d}\right)}^{2} \geq$ $a\|u\|_{L^{2}\left(D ; \mathbb{R}^{d}\right)}^{2}$ (see e.g. [13, Theorem 7.10, page 155]). From this inequality and (M) it follows that $\mathrm{A}$ is strictly monotone. Indeed, for any $u, v \in W_{0}^{1,2}\left(D ; \mathbb{R}^{d}\right)$, $u \neq v$, we have

$$
\begin{aligned}
\langle\mathrm{A} u-\mathrm{A} v, u-v\rangle & =\|\nabla(u-v)\|^{2}+\langle F(u)-F(v), u-v\rangle \\
& \geq\|\nabla(u-v)\|^{2}-L\|u-v\|^{2} \\
& \geq a\|u-v\|^{2}-L\|u-v\|^{2} \\
& =(a-L)\|u-v\|^{2}>0 .
\end{aligned}
$$

Using again Poincare's inequality, we have that

$$
\begin{aligned}
\langle\mathrm{A} u, u\rangle & =\|\nabla u\|^{2}+\langle F(u), u\rangle \\
& =\|\nabla u\|^{2}+\langle F(u)-F(0), u\rangle+\langle F(0), u\rangle \\
& \geq\|\nabla u\|^{2}-L\|u\|^{2}+\langle F(0), u\rangle \\
& \geq(a-L)\|u\|^{2}+\langle F(0), u\rangle .
\end{aligned}
$$

Then, since $\left|\frac{\langle F(0), u\rangle}{\|u\|}\right| \leq|F(0)|_{\mathbb{R}^{d}}$, we see that

$$
\lim _{\|u\|_{W_{0}^{1,2}\left(D ; \mathbb{R}^{d}\right)} \rightarrow+\infty} \frac{\langle A u, u\rangle}{\|u\|}=+\infty,
$$

proving coercivity.

For any $u, v, w \in W_{0}^{1,2}\left(D ; \mathbb{R}^{d}\right)$, we have

$$
\begin{aligned}
\langle A(u+t v), w\rangle & =\int_{D} \nabla u(x) \nabla w(x) d x+t \int_{D} \nabla v(x) \nabla w(x) d x \\
& +\int_{D} f(u(x)+t v(x)) w(x) d x .
\end{aligned}
$$

This yields the continuity of the mapping $t \mapsto\langle\mathrm{A}(u+t v), w\rangle$ on $[0,1]$. Thus, $\mathrm{A}$ is hemicontinuous. 
Therefore the equation $\mathrm{A} \omega=b_{n}$ has a unique solution on $W_{0}^{1,2}\left(D ; \mathbb{R}^{d}\right)$ that we denote by $u^{n}$, and the sequence $\left(u^{n}\right)_{n \geq 1}$ satisfies

$$
u^{n}(x)+\int_{D} G_{D}^{k}(x, y) f\left(u^{n}(y)\right) d y=b_{n}(x),
$$

for $x \in D$, and $\left.u^{n}\right|_{\partial D}=0$.

Step 2: Passing to the limit. We prove that $\left(u_{n}\right)_{n \geq 1}$ is a Cauchy sequence in $L^{2}\left(D ; \mathbb{R}^{d}\right)$. Indeed, fix $n, m \geq 1$. Starting with the identity

$$
u^{n}(x)-u^{m}(x)+\int_{D} G_{D}^{k}(x, y)\left[f\left(u^{n}(y)\right)-f\left(u^{m}(y)\right)\right] d y=b_{n}-b_{m},
$$

and taking the inner product with $F\left(u^{n}\right)-F\left(u^{m}\right)$ in $L^{2}\left(D ; \mathbb{R}^{d}\right)$, yields

$$
\begin{aligned}
\left\langle u^{n}\right. & \left.-u^{m}, F\left(u^{n}\right)-F\left(u^{m}\right)\right\rangle \\
& +\left\langle\int_{D} G_{D}^{k}(\cdot, y)\left(f\left(u^{n}(y)\right)-f\left(u^{m}(y)\right)\right) d y, F\left(u^{n}\right)-F\left(u^{m}\right)\right\rangle \\
& =\left\langle F\left(u^{n}\right)-F\left(u^{m}\right), b_{n}-b_{m}\right\rangle .
\end{aligned}
$$

The assumption $(\mathbf{M})$ and the property $(\mathbf{P})$ implies

$$
\begin{aligned}
-L\left\|u^{n}-u^{m}\right\|^{2} & +a\left\|\int_{D} G_{D}^{k}(\cdot, y)\left(f\left(u^{n}(y)\right)-f\left(u^{m}(y)\right)\right) d y\right\|^{2} \\
& \leq\left\langle F\left(u^{n}\right)-F\left(u^{m}\right), b_{n}-b_{m}\right\rangle .
\end{aligned}
$$

By substracting the expresion (10) for $n$ and $m$, respectively, we obtain,

$$
\begin{aligned}
\left\|\int_{D} G(\cdot, y)\left(f\left(u^{n}(y)\right)-f\left(u^{m}(y)\right)\right) d y\right\|^{2} & =\left\|u^{n}-u^{m}\right\|^{2}+\left\|b_{n}-b_{m}\right\|^{2} \\
& -2\left\langle u^{n}-u^{m}, b_{n}-b_{m}\right\rangle .
\end{aligned}
$$

Multiplying this identity by $a$ and using (11), we have

$$
\begin{aligned}
& (a-L)\left\|u^{n}-u^{m}\right\|^{2}+a\left\|b_{n}-b_{m}\right\|^{2} \\
& \quad \leq\left\langle F\left(u^{n}\right)-F\left(u^{m}\right)+2 a\left(u^{n}-u^{m}\right), b_{n}-b_{m}\right\rangle \\
& \quad \leq\left\|b_{n}-b_{m}\right\|\left(\left\|F\left(u^{n}\right)-F\left(u^{m}\right)\right\|+2 a\left\|u^{n}-u^{m}\right\|\right) .
\end{aligned}
$$

We are assuming $0<L<a$. Hence we conclude that $\lim _{n, m \rightarrow \infty}\left\|u^{n}-u^{m}\right\|=0$.

Let $u$ be the $L^{2}\left(D ; \mathbb{R}^{d}\right)$-limit of the sequence $\left(u^{n}\right)_{n \geq 1}$. Applying first Hölder's inequality with respect to the measure $G(\cdot, y) d y$ and then Fubini's Theorem, we 
obtain:

$$
\begin{aligned}
\| \int_{D} & G_{D}^{k}(\cdot, y)\left(f\left(u^{n}(y)\right)-f(u(y))\right) d y \|^{2} \\
\quad & =\int_{D}\left(\int_{D} G_{D}^{k}(x, y)\left(f\left(u^{n}(y)\right)-f(u(y))\right) d y\right)^{2} d x \\
& \leq C \int_{D} \int_{D} G_{D}^{k}(x, y)\left(f\left(u^{n}(y)\right)-f(u(y))\right)^{2} d y d x \\
& =C \int_{D}\left(f\left(u^{n}(y)\right)-f(u(y))\right)^{2}\left(\int_{D} G_{D}^{k}(x, y) d x\right) d y \\
& \leq C \int_{D}\left(f\left(u^{n}(y)\right)-f(u(y))\right)^{2} d y .
\end{aligned}
$$

Since the operator F is strongly continuous, this yields

$$
\left\|\int_{D} G_{D}^{k}(\cdot, y)\left(f\left(u^{n}(y)\right)-f(u(y))\right) d y\right\|_{n \rightarrow \infty}^{2} \underset{n}{\longrightarrow}
$$

Let $b \in \mathcal{S}$ be given by

$$
b(x)=\int_{D} G_{D}^{k}(x, y) g^{i}(y) d y+\sigma \int_{D} G_{D}^{k}(x, y) W(d y) .
$$

Consider a sequence $\left(b_{n}\right)_{n \geq 1} \in \mathcal{C}_{0}^{\infty}\left(D ; \mathbb{R}^{d}\right)$, such that $b_{n} \rightarrow b$ in $L^{2}\left(D ; \mathbb{R}^{d}\right)$. By taking limits in the $L^{2}\left(D ; \mathbb{R}^{d}\right)$-norm in 100 , we obtain that a.s., $u$ satisfies the system of equations defined in (6) on $L^{2}\left(D ; \mathbb{R}^{d}\right)$. By continuity, for almost all $\omega \in \Omega$, this is also an identity for any $x \in D$.

\section{Properties of the solution}

In this section we study the existence of moments of the solution to (6) and also the Hölder continuity of its sample paths. For this, we need a slightly stronger assumption than $\mathbf{M}$, as follows.

$(\overline{\mathbf{M}})$ The operator $F: L^{2}\left(D ; \mathbb{R}^{d}\right) \rightarrow L^{2}\left(D ; \mathbb{R}^{d}\right)$ defined in Section 2 admits a decomposition $F=F_{1}+F_{2}$ which satisfies:

(a) $F_{1}: L^{2}\left(D ; \mathbb{R}^{d}\right) \rightarrow L^{2}\left(D ; \mathbb{R}^{d}\right)$ is bounded. Moreover, for all $v, w \in L^{2}\left(D ; \mathbb{R}^{d}\right)$,

$$
\left\langle v-w, F_{1}(v)-F_{1}(w)\right\rangle \geq 0
$$

(b) $F_{2}: L^{2}\left(D ; \mathbb{R}^{d}\right) \rightarrow L^{2}\left(D ; \mathbb{R}^{d}\right)$ is such that there exists $L>0$ and for any $v \in L^{2}\left(D ; \mathbb{R}^{d}\right)$,

$$
\left|F_{2}(v)\left(z_{1}\right)-F_{2}(v)\left(z_{2}\right)\right| \leq L\left|v\left(z_{1}\right)-v\left(z_{2}\right)\right|, \forall z_{1}, z_{2} \in \mathbb{R}^{d} .
$$


If $d=1$, the assumptions $\mathbf{M}$ and $(\overline{\mathbf{M}})$ are equivalent. In general, $(\overline{\mathbf{M}})$ implies $\mathbf{M}$ (with the same constant $L$ ).

Proposition 3.1 The hypotheses are

(i) $g \in L^{2}\left(D ; \mathbb{R}^{d}\right)$;

(ii) The operator F satisfies the conditions $\boldsymbol{C}$ and $(\overline{\boldsymbol{M}})$;

(iii) Let $K=\sup _{x \in D}\left\|G_{D}^{k}(x, \cdot)\right\|_{L^{2}(D)}$. The constants $L$ and a in $(\overline{\boldsymbol{M}})$ and $(\boldsymbol{P})$ respectively, satisfy $0<L<a \wedge\left(K|D|^{\frac{1}{2}}\right)^{-1}$, where $|D|$ denotes the Lebesgue measure of $D$.

Then for all $p \geq 2$,

$$
\|u\|_{L^{p}\left(\Omega ; L^{2}\left(D ; \mathbb{R}^{d}\right)\right)}<\infty .
$$

Proof: From (6) and the triangular inequality, we have

$$
\|u\|_{L^{p}\left(\Omega ; L^{2}(D)\right)} \leq S_{1}+S_{2}+S_{3},
$$

with

$$
\begin{aligned}
& S_{1}=\left\|\int_{D} G_{D}^{k}(\cdot, y) f(u(y)) d y\right\|_{L^{p}\left(\Omega ; L^{2}\left(D ; \mathbb{R}^{d}\right)\right)}, \\
& S_{2}=\left\|\int_{D} G_{D}^{k}(\cdot, y) g(y) d y\right\|_{L^{2}\left(D ; \mathbb{R}^{d}\right)}, \\
& S_{3}=\left\|\int_{D} G_{D}^{k}(\cdot, y) W(d y)\right\|_{L^{p}\left(\Omega ; L^{2}\left(D ; \mathbb{R}^{d}\right)\right)} .
\end{aligned}
$$

Let $f_{j}: \mathbb{R}^{d} \longrightarrow \mathbb{R}^{d}$ be defined as $f_{j}(v(y))=F_{j}(v)(y)$, for any $v \in L^{2}\left(D ; \mathbb{R}^{d}\right)$, $y \in \mathbb{R}^{d}, j=1,2$. Then

$$
\begin{aligned}
S_{1} & \leq\left\|\int_{D} G_{D}^{k}(\cdot, y) f_{1}(u(y)) d y\right\|_{L^{p}\left(\Omega ; L^{2}\left(D ; \mathbb{R}^{d}\right)\right)} \\
& +\left\|\int_{D} G_{D}^{k}(\cdot, y) f_{2}(u(y)) d y\right\|_{L^{p}\left(\Omega ; L^{2}\left(D ; \mathbb{R}^{d}\right)\right)} .
\end{aligned}
$$

For the first term on the right-hand side of this inequality we have,

$$
\begin{aligned}
\| \int_{D} & G_{D}^{k}(\cdot, y) f_{1}(u(y)) d y \|_{L^{p}\left(\Omega ; L^{2}\left(D ; \mathbb{R}^{d}\right)\right)} \\
& =\left(\mathbb{E}\left(\int_{D} d x\left|\int_{D} G_{D}^{k}(x, y) f_{1}(u(y)) d y\right|^{2}\right)^{p / 2}\right)^{1 / p} \\
& \leq\left(\mathbb{E}\left(\int_{D} d x\left(\int_{D} G_{D}^{k}(x, y)^{2} d y\right) \int_{D}\left|f_{1}(u(y))\right|^{2} d y\right)^{p / 2}\right)^{1 / p} \\
& \leq M|D|^{1 / 2} \sup _{x \in D}\left\|G_{D}^{k}(x, \cdot)\right\| .
\end{aligned}
$$


For the second one, we use property (b) of $(\overline{\mathbf{M}})$ to obtain

$$
\begin{aligned}
& \left\|\int_{D} G_{D}^{k}(\cdot, y) f_{2}(u(y)) d y\right\|_{L^{p}\left(\Omega ; L^{2}\left(D ; \mathbb{R}^{d}\right)\right)} \\
& \quad \leq\left\|\int_{D} G_{D}^{k}(\cdot, y) f_{2}(0) d y\right\|_{L^{p}\left(\Omega ; L^{2}\left(D ; \mathbb{R}^{d}\right)\right)} \\
& \quad+\left\|\int_{D} G_{D}^{k}(\cdot, y)\left(f_{2}(u(y))-f_{2}(0)\right) d y\right\|_{L^{p}\left(\Omega ; L^{2}\left(D ; \mathbb{R}^{d}\right)\right)} \\
& \quad \leq\left|f_{2}(0)\right| \sup _{x \in D}\left\|G_{D}^{k}(x, \cdot)\right\||| D \mid \\
& \quad+L \sup _{x \in D}\left\|G_{D}^{k}(x, \cdot)\right\||D|^{1 / 2}\|u\|_{L^{p}\left(\Omega ; L^{2}\left(D ; \mathbb{R}^{d}\right)\right)} .
\end{aligned}
$$

By applying Schwarz's inequality, we have

$$
\begin{aligned}
& S_{2}=\left(\int_{D} d x\left|\int_{D} G_{D}^{k}(x, y) g(y) d y\right|^{2}\right)^{1 / 2} \\
& \leq \sup _{x \in D}\left\|G_{D}^{k}(x, \cdot)\right\|_{L^{2}(D)}|D|^{1 / 2}\|g\| .
\end{aligned}
$$

Finally, we study $S_{3}$. We apply first Hölder's inequality with respect to the Lebesgue measure $d x$, then the hypercontractivity property of Gaussian randon vectors and finally, the isometry property of the stochastic integral. This yields

$$
\begin{aligned}
S_{3} & =\left(\mathbb{E}\left(\int_{D} d x\left|\int_{D} G_{D}^{k}(x, y) W(d y)\right|^{2}\right)^{p / 2}\right)^{1 / p} \\
& \leq|D|^{1 / 2-1 / p}\left(\mathbb{E}\left(\int_{D} d x\left|\int_{D} G_{D}^{k}(x, y) W(d y)\right|^{p}\right)\right)^{1 / p} \\
& \leq C_{p}|D|^{1 / 2-1 / p}\left(\int_{D} d x\left(\mathbb{E}\left|\int_{D} G_{D}^{k}(x, y) W(d y)\right|^{2}\right)^{p / 2}\right)^{1 / p} \\
& \leq C_{p}|D|^{1 / 2} \sup _{x \in D}\left\|G_{D}^{k}(x, \cdot)\right\| .
\end{aligned}
$$

By Lemma 2.1, $K=\sup _{x \in D}\left\|G_{D}^{k}(x, \cdot)\right\|_{L^{2}(D)}$ is finite. Hence, from the upper bounds proved so far we infer that

$$
\|u\|_{L^{p}\left(\Omega ; L^{2}(D)\right)} \leq C_{1}+L K|D|^{\frac{1}{2}}\|u\|_{L^{p}\left(\Omega ; L^{2}(D)\right)},
$$

with $C_{1}=K|D|^{\frac{1}{2}}\left[M+\left|f_{2}(0)\right||D|^{\frac{1}{2}}+\|g\|+C_{p}\right]$. Since we are assuming $1-$ $L K|D|^{\frac{1}{2}}>0$, this yields the Proposition. 
Remark 3.2 In the context of elliptic operators, the assumption on the constant $L$ in the preceding Proposition is natural. It is a restriction to preserve the positiveness property of the operator $-\Delta+F$.

Proposition 3.3 The hypotheses are as in Proposition 3.1 Fix a ball centered at 0 and with radius $r, B_{r}(0)$, strictly included in $D$. Then, for any $p \geq 2$ there exists $a$ positive constant $C$ (depending on $r$ ) such that, for any $x_{1}, x_{2} \in B_{r}(0)$,

$$
\mathbb{E}\left[\left|u\left(x_{1}\right)-u\left(x_{2}\right)\right|^{p}\right] \leq C\left|x_{1}-x_{2}\right|^{p \xi},
$$

with

$$
\xi= \begin{cases}1, & \text { if } k=1, \\ 1-\gamma, & \text { if } k=2, \\ \frac{1}{2}, & \text { if } k=3,\end{cases}
$$

where $\gamma>0$ is arbitrarily small. Therefore, for almost all $\omega \in \Omega$, the sample paths of the process $u$ are Hölder continuous of degree $\alpha \in(0,1)$, if $k=1,2$, and $\alpha \in(0,1 / 2)$, if $k=3$.

Proof: From (6), we clearly have

$$
\mathbb{E}\left[\left|u\left(x_{1}\right)-u\left(x_{2}\right)\right|^{p}\right] \leq C_{p}\left(A\left(x_{1}, x_{2}\right)+B\left(x_{1}, x_{2}\right)+C\left(x_{1}, x_{2}\right)\right),
$$

where

$$
\begin{aligned}
& A\left(x_{1}, x_{2}\right)=\mathbb{E}\left[\left|\int_{D}\left[G_{D}^{k}\left(x_{1}, y\right)-G_{D}^{k}\left(x_{2}, y\right)\right] f(u(y)) d y\right|^{p}\right], \\
& B\left(x_{1}, x_{2}\right)=\mathbb{E}\left[\left|\int_{D}\left[G_{D}^{k}\left(x_{1}, y\right)-G_{D}^{k}\left(x_{2}, y\right)\right] g(y) d y\right|^{p}\right], \\
& C\left(x_{1}, x_{2}\right)=\mathbb{E}\left[\left|\int_{D}\left[G_{D}^{k}\left(x_{1}, y\right)-G_{D}^{k}\left(x_{2}, y\right)\right] W(d y)\right|^{p}\right] .
\end{aligned}
$$

The hypothesis $(\overline{\mathbf{M}})$ implies the following:

$$
\begin{aligned}
\int_{D}|f(u(y))|^{2} d y & \leq 2 M^{2}+2 \int_{D}\left|f_{2}(u(y))\right|^{2} d y \\
& \leq 2 M^{2}+4\left|D\left\|\left.f_{2}(0)\right|^{2}+4 L\right\| u \|^{2} .\right.
\end{aligned}
$$

Therefore,

$$
\mathbb{E}\left[\int_{D}|f(u(y))|^{2} d y\right]^{p / 2} \leq C_{1}\left(M,|D|, f_{2}(0), p\right)+C_{2}(L, p) \mathbb{E}\left[\|u\|^{p}\right],
$$

and the right-hand side of this expression is finite, due to Proposition 3.1 . 
Using this result and after applying Cauchy-Schwarz inequality, we obtain

$$
\begin{aligned}
A\left(x_{1}, x_{2}\right) & \leq\left[\int_{D}\left[G_{D}^{k}\left(x_{1}, y\right)-G_{D}^{k}\left(x_{2}, y\right)\right]^{2} d y\right]^{p / 2} \mathbb{E}\left[\int_{D}|f(u(y))|^{2} d y\right]^{p / 2} \\
& \leq C\left[\int_{D}\left[G_{D}^{k}\left(x_{1}, y\right)-G_{D}^{k}\left(x_{2}, y\right)\right]^{2} d y\right]^{p / 2}
\end{aligned}
$$

Applying again Cauchy-Schwarz inequality and using the properties of $g$ gives

$$
B\left(x_{1}, x_{2}\right) \leq C\left[\int_{D}\left[G_{D}^{k}\left(x_{1}, y\right)-G_{D}^{k}\left(x_{2}, y\right)\right]^{2} d y\right]^{p / 2} .
$$

Finally, the hypercontractivity property yields

$$
\begin{aligned}
C\left(x_{1}, x_{2}\right) & \leq C\left(E\left|\int_{D}\left[G_{D}^{k}\left(x_{1}, y\right)-G_{D}^{k}\left(x_{2}, y\right)\right] W(d y)\right|^{2}\right)^{p / 2} \\
& =C\left(\int_{D}\left|G_{D}^{k}\left(x_{1}, y\right)-G_{D}^{k}\left(x_{2}, y\right)\right|^{2} d y\right)^{p / 2}
\end{aligned}
$$

From (15)-(17) we see that

$$
\mathbb{E}\left[\left|u\left(x_{1}\right)-u\left(x_{2}\right)\right|^{p}\right] \leq C\left(\int_{D}\left|G_{D}^{k}\left(x_{1}, y\right)-G_{D}^{k}\left(x_{2}, y\right)\right|^{2} d y\right)^{p / 2} .
$$

We conclude the proof of (13) by applying lemmas 5.4, 5.5, 5.7 of Section 5.3, The statement on the sample paths of $u$ follows from Kolmogorov's continuity lemma.

Proposition 3.4 The hypotheses are as in Proposition 3.1 Then, for any $p \geq 2$,

$$
\sup _{x \in D}\|u(x)\|_{L^{p}\left(\Omega ; \mathbb{R}^{d}\right)}<\infty .
$$

Proof: It is similar to that of the preceding proposition. By the triangular inequality,

$$
\mathbb{E}\left[|u(x)|^{p}\right] \leq C_{p}(A(x)+B(x)+C(x)),
$$

with

$$
\begin{aligned}
& A(x)=\mathbb{E}\left[\left|\int_{D} G_{D}^{k}(x, y) f(u(y)) d y\right|^{p}\right], \\
& B(x)=\mathbb{E}\left[\left|\int_{D} G_{D}^{k}(x, y) g(y) d y\right|^{p}\right], \\
& C(x)=\mathbb{E}\left[\left|\int_{D} G_{D}^{k}(x, y) W(d y)\right|^{p}\right] .
\end{aligned}
$$


The conclusion will be obtained by proving that each of the above expressions are finite, uniformly in $x \in D$. This relies on Lemma 2.1 and the following arguments.

Applying (14) and Proposition 3.1yields

$$
\mathbb{E}\left[\int_{D}|f(u(y))|^{2} d y\right]^{p / 2} \leq C .
$$

Hence, by Cauchy-Schwarz inequality and Lemma2.1,

$$
A(x) \leq\left[\int_{D}\left[G_{D}^{k}(x, y)\right]^{2} d y\right]^{p / 2} \mathbb{E}\left[\int_{D}|f(u(y))|^{2} d y\right]^{p / 2} \leq C .
$$

Similarly,

$$
B(x) \leq C\left[\int_{D}\left[G_{D}^{k}(x, y)\right]^{2} d y\right]^{p / 2} \leq C .
$$

Finally, by the hypercontractivity property,

$$
\begin{aligned}
C(x) & \leq C\left(E\left|\int_{D} G_{D}^{k}(x, y) W(d y)\right|^{2}\right)^{p / 2} \\
& =C\left(\int_{D}\left|G_{D}^{k}(x, y)\right|^{2} d y\right)^{p / 2} \\
& \leq C .
\end{aligned}
$$

In all the expressions above, $C$ denotes a finite constant. Hence 180 ) holds.

\section{The law of the solution}

This section is devoted to prove that the probability law of the solution to the system of SPDEs (6) is absolutely continuous with respect to a Gaussian measure defined on the Banach space $\mathcal{S}$. As a consequence, for any fixed $x \in D$, the law of $u(x)$ is absolutely continuous with respect to the Lebesgue measure on $\mathbb{R}^{d}$. To a large extent, the content of the section is an extension to the $d$-dimensional case of results proved in [11].

Denote by $\mu$ the law on $\mathcal{S}$ of the Gaussian stochastic process

$$
\left(w(x)=\sigma \int_{D} G_{D}^{k}(x, y) W(d y), x \in D\right),
$$

and by $H$ the Hilbert space $L^{2}\left(D ; \mathbb{R}^{d}\right)$. Then, extending [11, Proposition 3.1] for $d=1$, we have that $(\mathcal{S}, H, \mu)$ is an abstract Wiener space. Indeed, $\mathcal{S}$ endowed with the supremum norm is a separable Banach space. By applying Schwarz inequality and then Lemma2.1, we obtain that the mapping

$$
\begin{aligned}
i: H & \longrightarrow \mathcal{S} \\
h & \mapsto \int_{D} G_{D}^{k}(\cdot, y) h(y) d y
\end{aligned}
$$


is continuous. Moreover, since the Dirichlet problem $\Delta v=h$ on $D, v \mid \partial D=0$, has a unique solution, we have that the mapping $i$ is one-to-one and clearly, $i(H)$ is densely embedded in $\mathcal{S}$.

For its further use throughout this section, we introduce a new assumption.

(I) The function $f$ is continuously differentiable, and $\operatorname{det} J_{f}(x) \neq 0$, for any $x \in$ $\mathbb{R}^{d}$, where $J_{f}$ denotes the Jacobian matrix of $f$. Moreover, the linear operator $J_{f}^{-1}(\omega): H \rightarrow H$ defined by $J_{f}^{-1}(\omega)(h)(x)=J_{f}^{-1}(\omega(x)) h(x)$ is positive, that is,

$$
\left\langle J_{f}^{-1}(\omega)(h), h\right\rangle>0, \forall h \in H .
$$

If $d=1$, the assumption is $f \in \mathcal{C}^{1}, f^{\prime}>0$ (see [11, (H.1), p. 229]).

Proposition 4.1 We keep the assumptions of Theorem 2.2 and in addition, we suppose that (I) holds. Then, the mapping

$$
\begin{aligned}
\bar{F}: \mathcal{S} & \longrightarrow i(H) \\
\omega & \mapsto i(F(\omega))=\int_{D} G_{D}^{k}(\cdot, y) f(\omega(y)) d y,
\end{aligned}
$$

satisfies the following properties.

1. For any $\omega \in \mathcal{S}$, there exists a Hilbert-Schmidt operator $D \bar{F}(\omega): H \longrightarrow H$ such that

$$
\|\bar{F}(\omega+i(h))-\bar{F}(\omega)-D \bar{F}(\omega)(h)\|_{H}=o\left(\|h\|_{H}\right), \text { as }\|h\|_{H} \rightarrow 0 .
$$

2. For any $\omega \in \mathcal{S}$, the mapping $h \mapsto D \bar{F}(\omega+i(h))$ is continuous from $H$ into the space of Hilbert-Schmidt operators on $H$.

3. For any $\omega \in \mathcal{S}$, the mapping $I_{H}+D \bar{F}(\omega)$ is invertible, where $I_{H}$ denotes the identity operator on $H$.

Proof: For any $\omega \in \mathcal{S}$, set

$$
D \bar{F}(\omega)(h)=J_{f}(\omega(\cdot)) \int_{D} G_{D}^{k}(\cdot, y) h(y) d y, h \in H .
$$

The assumptions on $f$ imply that $\int_{D}\left|J_{f}(\omega(x))\right|^{2} d x<\infty$. Then, by the definition of the Hilbert-Schmidt norm (see e.g. [20, Theorem VI.23, p. 210]) and by using Lemma2.1 we obtain,

$$
\begin{aligned}
\|D \bar{F}(\omega)\|_{H S}^{2} & =\int_{D} \int_{D}\left|J_{f}(\omega(x)) G_{D}^{k}(x, y)\right|^{2} d x d y \\
& \leq \sup _{x \in D}\left(\int_{D}\left(G_{D}^{k}(x, y)\right)^{2} d y\right)\left(\int_{D}\left|J_{f}(\omega(x))\right|^{2} d x\right)<\infty .
\end{aligned}
$$


This yields that $D \bar{F}(\omega)$ is a Hilbert-Schmidt operator.

From the expression (20), one checks that (19) is satisfied. Moreover, from assertion 1 . and the continuity of the map $J_{f}(\cdot)$, it is easy to verify that statement 2. holds.

For the proof of the third statement, we notice that the operator $D \bar{F}(\omega)$ is compact. Hence, by the Fredholm alternative it suffices to check that $\lambda=-1$ is not an eigenvalue. This fact is a consequence of the assumption (I). Indeed, if $\lambda=-1$ were an eigenvalue, there would exists a non null $h \in H$ satisfying

$$
h+J_{f}(\omega) i(h)=0 .
$$

Equivalently,

$$
J_{f}^{-1}(\omega) h+\int_{D} G_{D}^{k}(\cdot, y) h(y) d y=0 .
$$

Take the inner product in $H$ with $h$ on each term of this identity. By property $(\mathbf{P})$, we obtain

$$
\left\langle J_{f}^{-1}(\omega) h, h\right\rangle+a\left\|\int_{D} G_{D}^{k}(\cdot, y) h(y) d y\right\|^{2}=0 .
$$

By assumption (I), this implies that $h=0$.

In terms of $\bar{F}$, the operator $\mathrm{T}$ defined in $(8)$ is $\mathrm{T}=I_{B}+\bar{F}$. Hence, Proposition 4.1 tell us that $\mathrm{T}$ satisfies the assumptions of [15, Theorem 6.4]. This yields the following result

Proposition 4.2 The hypotheses are as in Proposition 4.1 Denote by $\nu$ the law of $u=\mathrm{T}^{-1}(w)$. Then, the probability $\nu$ is absolutely continuous with respect to $\mu$ (the law of $w$ ). Moreover, the density is given by

$$
\frac{d \nu}{d \mu}(\omega)=\left|\operatorname{det}_{2}\left(I_{H}+D \bar{F}(\omega)\right)\right| \exp \left(-\delta(f(\omega))-\frac{1}{2}\|f(\omega)\|_{H}^{2}\right),
$$

where det $_{2}$ denotes the Carleman-Fredholm determinant, and $\delta$ denotes the divergence operator, also called the Skorohod integral operator (see [2] Theorem 5.8.3] for a definition of this notion in this context).

Remark 4.3 For any $x \in D$, let $\pi_{x}: \mathcal{S} \longrightarrow \mathbb{R}^{d}$ be defined by $\pi_{x}(\omega)=\omega(x)$. Clearly, $\nu \ll \mu$ implies $\nu \circ \pi_{x}^{-1} \ll \mu \circ \pi_{x}^{-1}$. Since $\mu \circ \pi_{x}^{-1}$ is the law of the random vector $w(x)$, which is Gaussian, we infer that the law of $u(x)$ is absolutely continuous with respect to the Lebesgue measure on $\mathbb{R}^{d}$.

\section{Gaussian solutions}

In this section we consider the system (6) in the particular case $f=g=0$. Under this assumption, (6) gives an explicit expression of the solution, which clearly defines the $d$-dimensional Gaussian random vector: 


$$
w^{i}(x)=\sum_{j=1}^{d} \sigma_{i j} \int_{D} G_{D}^{k}(x, y) W^{j}(d y), x \in D, i=1, \ldots, d .
$$

We are assuming that $\sigma=\left(\sigma_{i j}\right)_{1 \leq i, j \leq d}$ is a non-singular matrix. Therefore, without loss of generality, we can reduce the analysis of the stochastic process given in (22) to the case where $\sigma$ is the identity matrix in $\mathbb{R}^{d}$. By doing so, we are left to consider the Gaussian vector $v(x)=\left(v^{i}(x)\right)_{i}$ with independent, identically distributed components defined by

$$
v^{i}(x)=\int_{D} G_{D}^{k}(x, y) W^{i}(d y), x \in D, i=1, \ldots, d .
$$

Its density is given by the formula

$$
p_{v(x)}(z)=\left(2 \pi \sigma_{x}^{2}\right)^{-\frac{d}{2}} \exp \left(-\frac{|z|^{2}}{2 \sigma_{x}^{2}}\right), z \in \mathbb{R}^{d},
$$

where $\sigma_{x}=\left\|G_{D}^{k}(x, \cdot)\right\|$.

According to Corollary 5.8, the mapping $x \in D \mapsto \sigma_{x}$ is continuous and therefore, $\inf _{x \in K} \sigma_{x}$ and $\sup _{x \in K} \sigma_{x}$ are both achieved on any compact subset $K \subset$ $D$. Let $x_{0}, x_{1} \in K$ be such that

$$
0<\sigma_{x_{0}}=\inf _{x \in K} \sigma_{x} \leq \sup _{x \in K} \sigma_{x}=\sigma_{x_{1}}<\infty .
$$

Then,

$$
\sup _{(z, x) \in \mathbb{R}^{d} \times K} p_{v(x)}(z) \leq\left(2 \pi \sigma_{x_{0}}^{2}\right)^{-\frac{d}{2}}<\infty,
$$

and for any compact set $\tilde{K} \subset \mathbb{R}^{d}$,

$$
c_{1}\left(2 \pi \sigma_{x_{1}}^{2}\right)^{-\frac{d}{2}} \leq \inf _{(z, x) \in \tilde{K} \times K} p_{v(x)}(z),
$$

where $c_{1}=\inf _{z \in \tilde{K}} \exp \left(-\frac{|z|^{2}}{2 \sigma_{x_{0}}^{2}}\right)$.

\subsection{Sample paths of the process $v$}

From Theorem 3.3, we already know that the sample paths of the Gaussian process defined by (22) are Hölder continuous. However, under the standing assumptions, more can be said.

Case $k=1$

The trajectories of $\{v(x), x \in(0, b)\}$ are differentiable, a.s. Indeed, from the expression (2) and by applying the Itô formula we have,

$$
v^{i}(x)=\frac{x}{b} \int_{0}^{b} W^{i}(y) d y-\int_{0}^{x} W^{i}(y) d y, i=1, \ldots, d .
$$


(see [3, Lemma 2.1]).

Case $k=2,3$

Let $D=B_{1}(0)$ and $D_{0}=B_{\rho_{0}}(0)$ with $\rho_{0}<1$. For any $x, y \in D$ and $\gamma$ arbitrarily small, define

$$
\tau(x, y)= \begin{cases}|x-y|^{1-\gamma}, & \text { if } k=2, \\ |x-y|^{\frac{1}{2}}, & \text { if } k=3 .\end{cases}
$$

According to the discussion in [22, p. 164-167], and by applying the estimates (47), (49) (for $k=2$ ) and (59) (for $k=3$ ), we have the following results on the uniform modulus of continuity of the process $v$.

(1) Extensions of the classical Garsia-Rodemich-Rumsey Lemma (see [22, Theorems 4.1,4.2]) yield the existence of a random variable $A$ having moments of any order, such that, for any $x, y \in D_{0}$,

$$
|v(x)-v(y)| \leq A \tau(x, y) \sqrt{\log \left(1+\tau(x, y)^{-1}\right)} .
$$

(2) From results in [17], one can obtain more information on the random variable $A$. Indeed, there exists a constant $c>0$ such that

$$
\mathbb{E}\left\{\exp \left(c \sup _{x, y \in D_{0}} \frac{|v(x)-v(y)|^{2}}{\log \left(1+\tau(x, y)^{-1}\right)}\right)\right\}<\infty
$$

(see [22, Corollary 4.4]).

(3) By using entropy methods and the Gaussian isoperimetric inequality, we obtain

$$
\limsup _{|h| \rightarrow 0} \frac{\sup _{x \in D_{0}, y \in B_{h}(0)}|v(x+y)-v(x)|}{\tau(0, y) \sqrt{\log \left(1+\tau(0, y)^{-1}\right)}} \leq C,
$$

where $C$ is a finite positive constant. Whether this estimate is sharp is an open question.

Clearly, the above results yield Hölder continuity of the sample paths, a.s.

Let $k=2$. Using Lemma 5.5 and arguing as in [8, Chapter 5], we deduce the property:

For almost all $\omega$, the sample paths of the process $\left\{v(x), x \in D_{0}\right\}$ are Hölder continuous of degree $\alpha \in(0,1)$, though there are not Lipschitz continuous.

Similarly, for $k=3$, using Lemma 5.7 we have:

For almost all $\omega$, the sample paths of the process $\left\{v(x), x \in D_{0}\right\}$ are Hölder continuous of degree $\alpha \in(0,1 / 2)$, though there are not for $\alpha>1 / 2$. 


\subsection{Joint densities}

For $k=1, D_{0}$ denotes a closed interval of $D=(0, b)$, and as in the previous section, for $k=2,3, D_{0}=B_{\rho_{0}}(0)$, with $\rho_{0} \in(0,1)$. In this section we prove the following facts:

(a) $\operatorname{Var} v^{i}(x)>0$, for any $i=1, \ldots, d, x \in D_{0}$.

(b) $\operatorname{Corr}\left(v^{i}\left(x_{1}\right), v^{i}\left(x_{2}\right)\right)<1$, for any $i=1, \ldots, d$, and for each $x_{1}, x_{2} \in D_{0}$.

We recall that, for any $i=1, \ldots, d$,

$$
\operatorname{Var} v^{i}(x)=\left\|G_{D}^{k}(x, \cdot)\right\|^{2}:=\sigma_{x}^{2},
$$

and we will use the following notations:

$$
\begin{aligned}
\sigma_{x_{1}, x_{2}} & :=\operatorname{Cov}\left(v^{i}\left(x_{1}\right), v^{i}\left(x_{2}\right)\right)=\left\langle G_{D}^{k}\left(x_{1}, \cdot\right), G_{D}^{k}\left(x_{2}, \cdot\right)\right\rangle, \\
\rho_{x_{1}, x_{2}} & :=\operatorname{Corr}\left(v^{i}\left(x_{1}\right), v^{i}\left(x_{2}\right)\right)=\frac{\sigma_{x_{1}, x_{2}}}{\sigma_{x_{1}} \sigma_{x_{2}}} .
\end{aligned}
$$

Because of the independence of the components of $v\left(x_{1}\right)$ and of $v\left(x_{2}\right)$, properties (a) and (b) imply the existence of joint density of the $2 d$-dimensional vector

$$
\left(v\left(x_{1}\right), v\left(x_{2}\right)\right), x_{1}, x_{2} \in D_{0} .
$$

Property (a) follows trivially from (25). As for property (b), it is a consequence of property (a) and the next lemma.

Lemma 5.1 For any $x_{1}, x_{2} \in D_{0}, x_{1} \neq x_{2}$, we have

$$
\sigma_{x_{1}}^{2} \sigma_{x_{2}}^{2}-\sigma_{x_{1}, x_{2}}^{2}>0
$$

Proof: We argue by contradiction. Assume that $\sigma_{x_{1}}^{2} \sigma_{x_{2}}^{2}-\sigma_{x_{1}, x_{2}}^{2}=0$. Then, $\lambda \in$ $\mathbb{R} \backslash\{0\}$ (depending on $x_{1}, x_{2}$ ) would exist satisfying $v\left(x_{1}\right)=\lambda v\left(x_{2}\right)$. This implies $\left\|G_{D}^{k}\left(x_{1}, \cdot\right)-\lambda G_{D}^{k}\left(x_{2}, \cdot\right)\right\|=0$ or equivalently, $G_{D}^{k}\left(x_{1}, y\right)-\lambda G_{D}^{k}\left(x_{2}, y\right)=0$, for almost every $y$ (with respect to the Lebesgue measure).

Case $\lambda=1$. The condition $\left\|G_{D}^{k}\left(x_{1}, \cdot\right)-G_{D}^{k}\left(x_{2}, \cdot\right)\right\|=0$ yields a contradiction with the lower bounds given in (43), (49), (59), for $k=1, k=2, k=3$, respectively.

Case $\lambda \neq 1$. The condition $G_{D}^{k}\left(x_{1}, y\right)-\lambda G_{D}^{k}\left(x_{2}, y\right)=0$, for almost every $y$ implies that for any $f \in L^{2}(D)$,

$$
\left\langle G_{D}^{k}\left(x_{1}, y\right)-\lambda G_{D}^{k}\left(x_{2}, y\right), f\right\rangle=0 .
$$

Moreover,

$$
\left\|G_{D}^{k}\left(x_{1}, \cdot\right)\right\|=\lambda\left\|G_{D}^{k}\left(x_{2}, \cdot\right)\right\| .
$$


With this identity, and by developing the square of $\left\|G_{D}^{k}\left(x_{1}, \cdot\right)-\lambda G_{D}^{k}\left(x_{2}, \cdot\right)\right\|$, we obtain

$$
\lambda\left\|G_{D}^{k}\left(x_{2}, \cdot\right)\right\|^{2}=\left\langle G_{D}^{k}\left(x_{1}, \cdot\right), G_{D}^{k}\left(x_{2}, \cdot\right)\right\rangle .
$$

Choose $f=\nu G_{D}^{k}\left(x_{1}, \cdot\right)-G_{D}^{k}\left(x_{2}, \cdot\right)$, with $\nu \in \mathbb{R}$ to be determined later. The identity (33) implies,

$$
\begin{aligned}
0= & \left\langle G_{D}^{k}\left(x_{1}, \cdot\right)-\lambda G_{D}^{k}\left(x_{2}, \cdot\right), \nu G_{D}^{k}\left(x_{1}, \cdot\right)-G_{D}^{k}\left(x_{2}, \cdot\right)\right\rangle \\
= & \left\|G_{D}^{k}\left(x_{1}, \cdot\right)-G_{D}^{k}\left(x_{2}, \cdot\right)\right\|^{2}+(1-\lambda)\left\langle G_{D}^{k}\left(x_{1}, \cdot\right), G_{D}^{k}\left(x_{2}, \cdot\right)\right\rangle \\
& -(1-\lambda)\left\|G_{D}^{k}\left(x_{2}, \cdot\right)\right\|^{2}+(\nu-1)\left\|G_{D}^{k}\left(x_{1}, \cdot\right)\right\|^{2} \\
& -(\nu-1)\left\langle G_{D}^{k}\left(x_{1}, \cdot\right), G_{D}^{k}\left(x_{2}, \cdot\right)\right\rangle .
\end{aligned}
$$

By applying (34), (35) to (36) we obtain

$$
\begin{aligned}
0= & \left\langle G_{D}^{k}\left(x_{1}, \cdot\right)-\lambda G_{D}^{k}\left(x_{2}, \cdot\right), \nu G_{D}^{k}\left(x_{1}, \cdot\right)-G_{D}^{k}\left(x_{2}, \cdot\right)\right\rangle \\
= & \left\|G_{D}^{k}\left(x_{1}, \cdot\right)-G_{D}^{k}\left(x_{2}, \cdot\right)\right\|^{2} \\
& +(\lambda-1)[\lambda(\nu-2)+1]\left\|G_{D}^{k}\left(x_{2}, \cdot\right)\right\|^{2} .
\end{aligned}
$$

Assume first that $\lambda>1$. By choosing $\nu>2-\frac{1}{\lambda}$, the factor $(\lambda-1)[\lambda(\nu-2)+1]$ in (37) is positive. Hence, we obtain

$$
\begin{aligned}
0 & =\left\langle G_{D}^{k}\left(x_{1}, \cdot\right)-\lambda G_{D}^{k}\left(x_{2}, \cdot\right), \nu G_{D}^{k}\left(x_{1}, \cdot\right)-G_{D}^{k}\left(x_{2}, \cdot\right)\right\rangle \\
& \geq\left\|G_{D}^{k}\left(x_{1}, \cdot\right)-G_{D}^{k}\left(x_{2}, \cdot\right)\right\|^{2},
\end{aligned}
$$

which, arguing as for the case $\lambda=1$, yields a contradiction.

If $\lambda<1$, we choose $\nu<2-\frac{1}{\lambda}$ to obtain that $(\lambda-1)[\lambda(\nu-2)+1]>0$. Similarly as above, we arrive at a contradiction.

The proof of (32) is complete.

Lemma 5.2 Let $m_{x_{1}, x_{2}}=\frac{\sigma_{x_{1}, x_{2}}}{\sigma_{x_{1}}^{2}}$ be the conditional mean of $v^{i}\left(x_{2}\right)$ given $v^{i}\left(x_{1}\right)$, $i=1, \ldots, d$. Then, there exists a constant $C>0$ such that for all $x_{1}, x_{2} \in D_{0}$,

$$
\left|1-m_{x_{1}, x_{2}}\right| \leq C\left\|v\left(x_{1}\right)-v\left(x_{2}\right)\right\|_{L^{2}\left(\Omega ; \mathbb{R}^{d}\right)} .
$$

The proof of this lemma follows easily from the definition of $m_{x_{1}, x_{2}}$. We refer the reader to [9, p. 1359] for details.

Lemma 5.3 Let $\tau_{x_{1}, x_{2}}^{2}=\sigma_{x_{2}}^{2}\left(1-\rho_{x_{1}, x_{2}}^{2}\right)$ be the conditional variance of $v^{i}\left(x_{2}\right)$ given $v^{i}\left(x_{1}\right), i=1, \ldots, d$. Then, there exists a constant $C_{2}>0$ such that for all $x_{1}, x_{2} \in D_{0}$,

$$
\tau_{x_{1}, x_{2}} \leq C_{2}\left\|v\left(x_{1}\right)-v\left(x_{2}\right)\right\|_{L^{2}\left(\Omega ; \mathbb{R}^{d}\right)} .
$$

Proof: For any $x_{1}, x_{2} \in D$, let

$$
\delta\left(x_{1}, x_{2}\right):=\left\|v\left(x_{1}\right)-v\left(x_{2}\right)\right\|_{L^{2}\left(\Omega ; \mathbb{R}^{d}\right)}=\left\|G\left(x_{1}, \cdot\right)-G\left(x_{2}, \cdot\right)\right\|,
$$


be the canonical pseudo-metric associated with the Gaussian process $v$.

With simple computations, we obtain

$$
\sigma_{x_{2}}^{2} \sigma_{x_{1}}^{2}-\sigma_{x_{1}, x_{2}}^{2}=\frac{1}{4}\left[\delta\left(x_{1}, x_{2}\right)^{2}-\left(\sigma_{x_{2}}-\sigma_{x_{1}}\right)^{2}\right]\left[\left(\sigma_{x_{2}}+\sigma_{x_{1}}\right)^{2}-\delta\left(x_{1}, x_{2}\right)^{2}\right] .
$$

By the triangular inequality,

$$
\begin{aligned}
\left(\sigma_{x_{1}}-\sigma_{x_{2}}\right)^{2} & =\left|\left\|G\left(x_{1}, \cdot\right)\right\|-\left\|G\left(x_{2}, \cdot\right)\right\|\right|^{2} \\
& \leq\left\|G\left(x_{1}, \cdot\right)-G\left(x_{2}, \cdot\right)\right\|^{2}=\delta\left(x_{1}, x_{2}\right)^{2} .
\end{aligned}
$$

Hence, the first factor on the right-hand side of (41) is nonnegative. Moreover, we have proved in Lemma 5.1 that $1-\rho_{x_{1}, x_{2}}^{2}>0$. Hence, using [25], we have the following upper bounds:

$$
\begin{aligned}
1-\rho_{x_{1}, x_{2}}^{2} & \leq C\left[\delta\left(x_{1}, x_{2}\right)^{2}-\left(\sigma_{x_{2}}-\sigma_{x_{1}}\right)^{2}\right]\left(\sigma_{x_{2}}+\sigma_{x_{1}}\right)^{2} \\
& \leq C\left\{\delta\left(x_{1}, x_{2}\right)^{2}\left(\sigma_{x_{2}}^{2}+\sigma_{x_{1}}^{2}\right)+\left(\sigma_{x_{2}}^{2}-\sigma_{x_{1}}^{2}\right)^{2}\right\} \\
& \leq C\left[\delta\left(x_{1}, x_{2}\right)^{2}+\left(\sigma_{x_{1}}-\sigma_{x_{2}}\right)^{2}\right] \\
& \leq C \delta\left(x_{1}, x_{2}\right)^{2}
\end{aligned}
$$

The inequality (39) is a consequence of (25) and (42).

\subsection{Upper and lower bounds of the canonical metric}

In this section, we prove upper and lower bounds for the canonical pseudo-metric relative to the Gaussian process $v$ given in (40). This is equivalent to establish bounds from above and from below for $\left\|G_{D}^{k}\left(x_{1}, \cdot\right)-G_{D}^{k}\left(x_{2}, \cdot\right)\right\|$.

Lemma 5.4 Let $k=1$ and $D=(0, b), b>0$. For any $x_{1}, x_{2} \in D$, we have

$$
\left(\frac{b}{3}\right)^{\frac{1}{2}}\left|x_{1}-x_{2}\right| \leq\left\|G_{D}^{1}\left(x_{1}, \cdot\right)-G_{D}^{1}\left(x_{2}, \cdot\right)\right\| \leq\left(\frac{7 b}{3}\right)^{\frac{1}{2}}\left|x_{1}-x_{2}\right| .
$$

Proof: Using the expression (2), we clearly have

$$
\left\|G_{D}^{1}\left(x_{1}, \cdot\right)-G_{D}^{1}\left(x_{2}, \cdot\right)\right\|^{2}=\left|x_{1}-x_{2}\right|^{2} \int_{0}^{b}\left(\frac{x_{2} \wedge y-x_{1} \wedge y}{x_{2}-x_{1}}-\frac{y}{b}\right)^{2} d y .
$$

The integral on the right-hand side of this equality is $\frac{b}{3}+\frac{x_{2}^{2}+x_{2} x_{1}+x_{1}^{2}}{3 b}+x_{1}$. On $(0, b)$ this expression is bounded from above by $\frac{7 b}{3}$, and from below by $\frac{b}{3}$. This yields (43).

For $k=2,3, D=B_{1}(0)$, we will use the following formulas for the Green function (see for instance [13, [pg. 19]):

$$
\begin{aligned}
& G_{D}^{k}(x, y)=\Gamma^{k}\left((|x-y|)-\Gamma^{k}\left[|y|\left|x-\frac{y}{|y|^{2}}\right|\right], \quad y \neq 0,\right. \\
& G_{D}^{k}(x, y)=\Gamma^{k}(|x|)-\Gamma^{k}(1), \quad y=0 .
\end{aligned}
$$


with $\Gamma^{k}$ defined in (3).

For every $x, y \in D$, define

$$
L_{x}^{k}(y)=\Gamma^{k}(|x-y|), \quad S_{x}^{k}(y)=\Gamma^{k}\left[|y|\left|x-\frac{y}{|y|^{2}}\right|\right],
$$

so that for $y \neq 0$,

$$
G_{D}^{k}(x, y)=L_{x}^{k}(y)-S_{x}^{k}(y) .
$$

Notice that for any $y \in D, x \rightarrow S_{x}^{k}(y)$ is a harmonic function, and $S_{x}^{k}(y)=$ $L_{x}^{k}(y)$ for $y \in \partial D$.

Clearly, for any $x_{1}, x_{2} \in D$,

$$
\left\|G_{D}^{k}\left(x_{1}, \cdot\right)-G_{D}^{k}\left(x_{2}, \cdot\right)\right\| \leq\left\|L_{x_{1}}^{k}-L_{x_{2}}^{k}\right\|+\left\|S_{x_{1}}^{k}-S_{x_{2}}^{k}\right\| .
$$

Lemma 5.5 Let $k=2$ and $D=B_{1}(0)$. Fix $\rho_{0}<1$.

1. There exists a positive constant $C$ such that

$\left\|G_{D}^{2}\left(x_{1}, \cdot\right)-G_{D}^{2}\left(x_{2}, \cdot\right)\right\| \leq C\left|x_{1}-x_{2}\right|\left|\log ^{2}\left(\left|x_{1}-x_{2}\right|\right)-\log \left(\left|x_{1}-x_{2}\right|\right)+1\right|^{\frac{1}{2}}$,

for any $x_{1}, x_{2} \in \bar{B}_{\rho_{0}}(0)$. The constant $C$ above is of the form $\frac{c}{\left(1-\rho_{0}\right)^{2}}$ where $c>0$ is a multiple of $\pi^{-\frac{1}{2}}$.

Therefore,

$$
\begin{aligned}
& \left\|G_{D}^{2}\left(x_{1}, \cdot\right)-G_{D}^{2}\left(x_{2}, \cdot\right)\right\| \\
& \quad \leq C\left[\left|x_{1}-x_{2}\right|\left|\log \left(\left|x_{1}-x_{2}\right|\right)\right| 1_{\left\{\left|x_{1}-x_{2}\right| \leq e^{-1}\right\}}+\left|x_{1}-x_{2}\right| 1_{\left\{\left|x_{1}-x_{2}\right|>e^{-1}\right\}}\right],
\end{aligned}
$$

for any $x_{1}, x_{2} \in \bar{B}_{\rho_{0}}(0)$, where $C$ is a constant of the same type as in (47).

2. There exists a positive constant $\bar{C}$ such that

$$
\left\|G_{D}^{2}\left(x_{1}, \cdot\right)-G_{D}^{2}\left(x_{2}, \cdot\right)\right\| \geq \bar{C}\left|x_{1}-x_{2}\right|,
$$

for any $x_{1}, x_{2} \in \bar{B}_{\rho_{0}}(0)$. The constant $\bar{C}$ above is a multiple of $\pi^{-\frac{1}{2}}$.

Proof: First, we will prove an upper bound for $\left\|L_{x_{1}}^{2}-L_{x_{2}}^{2}\right\|_{2}$. Let $r_{x_{1}, x_{2}}=$ $2\left|x_{1}-x_{2}\right|$. Assume $\left|x_{1}-x_{2}\right|>1$. Then $\left|y-x_{1}\right| \leq r_{x_{1}, x_{2}}$, for any $|y| \leq 1$, and

$$
\left\|L_{x_{1}}^{2}-L_{x_{2}}^{2}\right\|^{2} \leq\left(2 \pi^{2}\right)^{-1}\left[J_{1}\left(x_{1}\right)+J_{2}\left(x_{2}\right)\right],
$$

with

$$
\begin{aligned}
& J_{1}\left(x_{1}\right)=\int_{\{|y| \leq 1\} \cap\left\{\left|y-x_{1}\right| \leq r_{x_{1}, x_{2}}\right\}} \log ^{2}\left|x_{1}-y\right| d y, \\
& J_{2}\left(x_{2}\right)=\int_{\{|y| \leq 1\} \cap\left\{\left|y-x_{2}\right| \leq \frac{\left.3 r_{x_{1}, x_{2}}\right\}}{2} \log ^{2}\left|x_{2}-y\right| d y .\right.}
\end{aligned}
$$


Using polar coordinates $(r, \theta)$ and a change of variables $\rho=r^{2}$, we have

$J_{1}\left(x_{1}\right) \leq \frac{\pi}{4} \int_{0}^{r_{x_{1}, x_{2}}^{2}}\left(\log ^{2} \rho\right) d \rho=\pi r_{x_{1}, x_{2}}^{2}\left(\log ^{2}\left[\frac{1}{r_{x_{1}, x_{2}}}\right]+\log \left[\frac{1}{r_{x_{1}, x_{2}}}\right]+\frac{1}{2}\right)$

where the integral is computed using integration by parts.

Similarly,

$$
J_{2}\left(x_{1}\right) \leq c r_{x_{1}, x_{2}}^{2}\left(\log ^{2}\left[\frac{1}{r_{x_{1}, x_{2}}}\right]+\log \left[\frac{1}{r_{x_{1}, x_{2}}}\right]+\frac{1}{2}\right),
$$

with a constant $\mathrm{c}$ which is a multiple of $\pi$ and, consequently

$$
\left\|L_{x_{1}}^{2}-L_{x_{2}}^{2}\right\|^{2} \leq C\left|x_{1}-x_{2}\right|^{2}\left(\log ^{2}\left[\frac{1}{\left|x_{1}-x_{2}\right|}\right]+\log \left[\frac{1}{\left|x_{1}-x_{2}\right|}\right]+1\right),
$$

for some positive constant $C$ which is a multiple of $\pi^{-1}$.

Next, we assume that $\left|x_{1}-x_{2}\right| \leq 1$. We have

$$
\left.\left\|L_{x_{1}}^{2}-L_{x_{2}}^{2}\right\|^{2} \leq \pi^{-2}\left[J_{1}\left(x_{1}\right)+J_{2}\left(x_{2}\right)\right)\right]+\left(2 \pi^{2}\right)^{-1} J_{3}\left(x_{1}, x_{2}\right),
$$

with

$$
J_{3}\left(x_{1}, x_{2}\right)=\int_{\left.\{|y| \leq 1\} \cap\left\{\left|y-x_{1}\right|>r_{x_{1}, x_{2}}\right\}\right\}}\left(\log \left|x_{1}-y\right|-\log \left|x_{2}-y\right|\right)^{2} d y .
$$

Let $\varphi(\lambda)=\log \left(\left|\lambda\left(x_{2}-y\right)+(1-\lambda)\left(x_{1}-y\right)\right|\right), \lambda \in(0,1)$. Then,

$$
\log \left|x_{2}-y\right|-\log \left|x_{1}-y\right|=\varphi(1)-\varphi(0)=\int_{0}^{1} \varphi^{\prime}(\lambda) d \lambda .
$$

Denote by $\alpha_{\lambda}$ the angle between the vectors $x_{1}-x_{2}$ and $\lambda\left(x_{2}-y\right)+(1-\lambda)\left(x_{1}-y\right)$. Direct computations show that

$$
\varphi^{\prime}(\lambda)=\frac{\left|x_{1}-x_{2}\right| \cos \left(\alpha_{\lambda}\right)}{\left|\lambda\left(x_{2}-y\right)+(1-\lambda)\left(x_{1}-y\right)\right|},
$$

Hence,

$$
\begin{aligned}
& J_{3}\left(x_{1}, x_{2}\right) \leq\left|x_{1}-x_{2}\right|^{2} \\
& \quad \times \int_{\left.\{|y| \leq 1\} \cap\left\{\left|y-x_{1}\right|>r_{x_{1}, x_{2}}\right\}\right\}} d y\left(\int_{0}^{1} \frac{1}{\left|\lambda\left(x_{2}-y\right)+(1-\lambda)\left(x_{1}-y\right)\right|} d \lambda\right)^{2} .
\end{aligned}
$$

On $\left\{\left|y-x_{1}\right|>r_{x_{1}, x_{2}}\right\}$,

$$
\left|\lambda\left(x_{2}-y\right)+(1-\lambda)\left(x_{1}-y\right)\right| \geq\left|y-x_{1}\right|-\lambda\left|x_{2}-x_{1}\right| \geq \frac{\left|y-x_{1}\right|}{2} .
$$


Therefore,

$$
\begin{aligned}
J_{3}\left(x_{1}, x_{2}\right) & \leq 4\left|x_{1}-x_{2}\right|^{2} \int_{\{|y| \leq 1\} \cap\left\{\left|y-x_{1}\right|>r_{x_{1}, x_{2}}\right\}}\left|y-x_{1}\right|^{-2} d y \\
& \leq 8 \pi\left|x_{1}-x_{2}\right|^{2} \log \left[\frac{1}{\left|x_{1}-x_{2}\right|}\right] .
\end{aligned}
$$

From (50)-(52), we have

$$
\left\|L_{x_{1}}^{2}-L_{x_{2}}^{2}\right\|^{2} \leq C\left|x_{1}-x_{2}\right|^{2}\left(\log ^{2} \frac{1}{\left|x_{1}-x_{2}\right|}+\log \frac{1}{\left|x_{1}-x_{2}\right|}+1\right),
$$

with a positive constant which is a multiple of $\pi^{-1}$.

For the study of the contribution of $\left\|S_{x_{1}}^{2}-S_{x_{2}}^{2}\right\|_{2}$ it is useful to identify $\mathbb{R}^{2}$ with $\mathbb{C}$ (the set of complex numbers) and to consider the following identity:

$$
|y|\left|x-\frac{y}{|y|^{2}}\right|=|1-\bar{x} y|,
$$

where $\bar{x}$ denotes the conjugate of the complex number $x$. By doing so, it is easy to check that

$$
2 \pi\left|\nabla_{x} S_{.}^{2}(y)\right|=\left|\nabla_{x} \log (|1-\bar{x} y|)\right| \leq \frac{|y|}{\sqrt{2}(1-|x|)^{2}} .
$$

By the mean value theorem, this implies,

$$
\begin{aligned}
\left\|S_{x_{1}}^{2}-S_{x_{2}}^{2}\right\|^{2} & =(2 \pi)^{-2} \int_{|y| \leq 1}|\log | 1-\bar{x}_{1} y|-\log | 1-\bar{x}_{2} y \|^{2} d y \\
& \leq\left(8 \pi^{2}\right)^{-1}\left|x_{1}-x_{2}\right|^{2} \int_{|y| \leq 1}\left(\frac{1}{\left(1-\left|x^{*}\right|\right)^{2}}\right)^{2} d y
\end{aligned}
$$

with $x^{*}=\lambda x_{1}+(1-\lambda) x_{2}$. We are assuming $x_{1}, x_{2} \in \bar{B}_{\rho_{0}}(0)$ with $\rho_{0}<1$. Hence, $1-\left|x^{*}\right| \geq 1-\rho_{0}$ and therefore,

$$
\left\|S_{x_{1}}^{2}-S_{x_{2}}^{2}\right\|^{2} \leq \frac{C}{\left(1-\rho_{0}\right)^{4}}\left|x_{1}-x_{2}\right|^{2},
$$

with a constant $\mathrm{C}$ which is a multiple of $\pi^{-1}$.

With (53), (56), we have proved (47).

If $\left|x_{1}-x_{2}\right| \leq e^{-1}$, then $\left|\log ^{2}\left(\left|x_{1}-x_{2}\right|\right)-\log \left(\left|x_{1}-x_{2}\right|\right)+1\right| \leq 3 \log ^{2}\left(\mid x_{1}-\right.$ $\left.x_{2} \mid\right)$, while if $\left|x_{1}-x_{2}\right|>e^{-1}$,

$$
\sup _{e^{-1}<\left|x_{1}-x_{2}\right| \leq 2}\left[\left|\log ^{2}\left(\left|x_{1}-x_{2}\right|\right)-\log \left(\left|x_{1}-x_{2}\right|\right)+1\right|\right] \leq C .
$$

Therefore (47) clearly implies (48). 
Next, we prove (49). Let $\eta \in\left(0, \frac{1-\rho_{0}}{2 \rho_{0}}\right)$. Since $\left|x_{1}-x_{2}\right| \leq 2 \rho_{0}$, we have $\eta\left|x_{1}-x_{2}\right|<1-\rho_{0}$. Let $D_{\eta}=\left\{y \in D:\left|y-x_{1}\right|<\eta\left|x_{1}-x_{2}\right|\right\}$. The choice of $\eta$ implies $D_{\eta} \subset D$, and then,

$$
\begin{aligned}
\left\|G_{D}^{2}\left(x_{1}, \cdot\right)-G_{D}^{2}\left(x_{2}, \cdot\right)\right\|^{2} & \geq\left\|G_{D}^{2}\left(x_{1}, \cdot\right)-G_{D}^{2}\left(x_{2}, \cdot\right)\right\|_{\eta}^{2} \\
& \geq \frac{1}{2}\left\|L_{x_{1}}^{2}-L_{x_{2}}^{2}\right\|_{\eta}^{2}-\left\|S_{x_{1}}^{2}-S_{x_{2}}^{2}\right\|_{\eta}^{2},
\end{aligned}
$$

where $\|\cdot\|_{\eta}$ denotes the $L^{2}$-norm on $D_{\eta}$. Similarly as in (55), using (54), se have

$$
\begin{aligned}
\left\|S_{x_{1}}^{2}-S_{x_{2}}^{2}\right\|_{\eta}^{2} & =\int_{D_{\eta}}\left(S_{x_{1}}^{2}(y)-S_{x_{2}}^{2}(y)\right)^{2} d y \\
& =\left|x_{1}-x_{2}\right|^{2} \int_{D_{\eta}}\left|\nabla_{x^{*}} S_{\cdot}^{2}(y)\right|^{2} d y \\
& \leq\left(8 \pi^{2}\right)^{-1}\left|x_{1}-x_{2}\right|^{2}\left(1-\rho_{0}\right)^{-4} \int_{D_{\eta}}|y|^{2} d y \\
& \leq c \pi^{-1}\left(1-\rho_{0}\right)^{-4} \eta^{2}\left|x_{1}-x_{2}\right|^{4} .
\end{aligned}
$$

We continue the proof by establishing a lower bound for $\left\|L_{x_{1}}^{2}-L_{x_{2}}^{2}\right\|_{\eta}^{2}$. For this, we take a new domain of integration $\bar{D}_{\eta} \subset D_{\eta}$ defined as the intersection of the set

$$
C_{\eta}=\left\{y \in D: \frac{\eta}{2}\left|x_{1}-x_{2}\right|<\left|y-x_{1}\right|<\eta\left|x_{1}-x_{2}\right|\right\}
$$

with the points $y \in D_{\eta}$ such that the angle between the lines joining $x_{1}$ with $y$ and $x_{1}$ with $x_{2}$ lies in the interval $(-\pi / 4, \pi / 4)$. Then, similarly as in the study of the term $J_{3}\left(x_{1}, x_{2}\right)$ above, we obtain

$$
\begin{aligned}
\left\|L_{x_{1}}^{2}-L_{x_{2}}^{2}\right\|_{\eta}^{2} \geq & (2 \pi)^{-2} \int_{\bar{D}_{\eta}}\left(\log \left|x_{1}-y\right|-\log \left|x_{2}-y\right|\right)^{2} d y \\
= & (2 \pi)^{-2}\left|x_{1}-x_{2}\right|^{2} \\
& \times \int_{\bar{D}_{\eta}}\left|\int_{0}^{1} \frac{\cos \left(\alpha_{\lambda}\right)}{\left|\lambda\left(x_{2}-y\right)+(1-\lambda)\left(x_{1}-y\right)\right|} d \lambda\right|^{2} d y .
\end{aligned}
$$

Remember that $\alpha_{\lambda}$ stands for the angle between the vectors $x_{1}-x_{2}$ and $\lambda\left(x_{2}-\right.$ $y)+(1-\lambda)\left(x_{1}-y\right)=x_{1}-y+\lambda\left(x_{2}-x_{1}\right)$. Also observe that, on $\bar{D}_{\eta}$, we have $1 / \sqrt{2} \leq \cos \left(\alpha_{\lambda}\right) \leq 1$, and $\left|y-\left[x_{1}+\lambda\left(x_{2}-x_{1}\right)\right]\right| \leq\left|y-x_{2}\right|$. Hence, from the above inequalities, we have

$$
\left\|L_{x_{1}}^{2}-L_{x_{2}}^{2}\right\|_{\eta}^{2} \geq\left(8 \pi^{2}\right)^{-1}\left|x_{1}-x_{2}\right|^{2} \int_{\bar{D}_{\eta}} \frac{d y}{\left|y-x_{2}\right|^{2}} .
$$

After the change of variables defined by $y \mapsto \frac{1}{2}\left(y-x_{2}\right)$ and then by using polar coordinates, we have

$$
\int_{\bar{D}_{\eta}} \frac{d y}{\left|y-x_{2}\right|^{2}}=C \pi \int_{\frac{\eta}{4}\left|x_{1}-x_{2}\right|}^{\frac{\eta}{2}\left|x_{1}-x_{2}\right|} \frac{d r}{r}=C \log 2 .
$$


Thus,

$$
\left\|L_{x_{1}}^{2}-L_{x_{2}}^{2}\right\|_{\eta}^{2} \geq C \pi^{-1}\left|x_{1}-x_{2}\right|^{2} .
$$

Along with (57) this yields

$$
\left\|G_{D}^{2}\left(x_{1}, \cdot\right)-G_{D}^{2}\left(x_{2}, \cdot\right)\right\|^{2} \geq C \pi^{-1}\left|x_{1}-x_{2}\right|^{2}\left(1-\left(1-\rho_{0}\right)^{-4} 4 \eta^{2}\right) .
$$

Finally, by choosing $\eta \in\left(0, \frac{\left(1-\rho_{0}\right)^{2}}{2 \sqrt{2}} \wedge \frac{1-\rho_{0}}{2 \rho_{0}}\right)$, we see that

$$
\left\|G_{D}^{2}\left(x_{1}, \cdot\right)-G_{D}^{2}\left(x_{2}, \cdot\right)\right\|^{2} \geq C \pi^{-1}\left|x_{1}-x_{2}\right|^{2},
$$

proving (49).

Remark 5.6 There is a gap between the upper and lower bounds in 487, (49), respectively, which means that at least the lower bound is not sharp. The consequences of this fact in the study of the hitting probabilities in Section 5.4 have been discussed in the introduction.

Lemma 5.7 Let $k=3$ and $D=B_{1}(0)$. Fix $\rho_{0}<1$. Then, there exist two positive constants

$$
\begin{aligned}
\tilde{C} & =\tilde{c}\left(\frac{1-\rho_{0}}{2 \rho_{0}} \wedge \frac{1}{19} \wedge\left(1-\rho_{0}\right)^{4}\right)^{\frac{1}{2}}, \\
C & =c \frac{1}{\left(1-\rho_{0}\right)^{2}},
\end{aligned}
$$

with $\tilde{c}$ and c some multiple of $\pi^{-1 / 2}$, such that for any $x_{1}, x_{2} \in B_{\rho_{0}}(0)$,

$$
\tilde{C}\left|x_{1}-x_{2}\right|^{\frac{1}{2}} \leq\left\|G_{D}^{3}\left(x_{1}, \cdot\right)-G_{D}^{3}\left(x_{2}, \cdot\right)\right\| \leq C\left|x_{1}-x_{2}\right|^{\frac{1}{2}} .
$$

Proof: We fix $x_{1}, x_{2} \in \bar{B}_{\rho_{0}}(0), x_{1} \neq x_{2}$, and start by proving the upper bound. For this, we first find a bound from above for $\left\|L_{x_{1}}^{3}-L_{x_{2}}^{3}\right\|_{2}$, using a similar approach as for $k=2$. Let $x_{1}, x_{2}$ be distinct points in $B_{\rho_{0}}(0)$ and set $r_{x_{1} x_{2}}=$ $2\left|x_{1}-x_{2}\right|$. Assume $\left|x_{1}-x_{2}\right|>1$. Then $\left|y-x_{1}\right| \leq r_{x_{1} x_{2}}$, for any $|y| \leq 1$, and

$$
\left\|L_{x_{1}}^{3}-L_{x_{2}}^{3}\right\|^{2} \leq\left(8 \pi^{2}\right)^{-1}\left[I_{1}\left(x_{1}\right)+I_{2}\left(x_{2}\right)\right],
$$

with

$$
\begin{aligned}
& I_{1}\left(x_{1}\right)=\int_{\{|y| \leq 1\} \cap\left\{\left|y-x_{1}\right| \leq r_{x_{1} x_{2}}\right\}}\left|x_{1}-y\right|^{-2} d y, \\
& I_{2}\left(x_{2}\right)=\int_{\{|y| \leq 1\} \cap\left\{\left|y-x_{2}\right| \leq \frac{\left.3 r_{x_{1} x_{2}}\right\}}{2}\left|x_{2}-y\right|^{-2} d y .\right.}
\end{aligned}
$$

Applying the change of variables given by the spherical coordinates yields

$$
I_{1}\left(x_{1}\right)+I_{2}\left(x_{2}\right) \leq 20 \pi\left|x_{1}-x_{2}\right|
$$


Next, we assume that $\left|x_{1}-x_{2}\right| \leq 1$. We have

$$
\left.\left\|L_{x_{1}}^{3}-L_{x_{2}}^{3}\right\|^{2} \leq\left(4 \pi^{2}\right)^{-1}\left[I_{1}\left(x_{1}\right)+I_{2}\left(x_{2}\right)\right)\right]+\left(8 \pi^{2}\right)^{-1} I_{3}\left(x_{1}, x_{2}\right),
$$

with

$$
I_{3}\left(x_{1}, x_{2}\right)=\int_{\left.\{|y| \leq 1\} \cap\left\{\left|y-x_{1}\right|>r_{x_{1} x_{2}}\right\}\right\}}\left(\frac{1}{\left|x_{1}-y\right|}-\frac{1}{\left|x_{2}-y\right|}\right)^{2} d y .
$$

A direct computation shows that $\left|\nabla_{x}\left(|\cdot-y|^{-1}\right)\right|=|x-y|^{-2}$. Using this fact, along with the mean value theorem, we obtain

$$
I_{3}\left(x_{1}, x_{2}\right) \leq\left|x_{1}-x_{2}\right|^{2} \int_{\left.\{|y| \leq 1\} \cap\left\{\left|y-x_{1}\right|>r_{x_{1} x_{2}}\right\}\right\}}\left|x^{*}-y\right|^{-4} d y,
$$

with $x^{*}=x_{1}+\lambda\left(x_{2}-x_{1}\right)$ for some $\lambda \in(0,1)$.

On the set $\left\{\left|y-x_{1}\right|>r_{x_{1} x_{2}}\right\}$,

$$
\begin{aligned}
\left|x^{*}-y\right| & =\left|x_{1}-y+\lambda\left(x_{2}-x_{1}\right)\right| \geq\left|x_{1}-y\right|-\lambda\left|x_{2}-x_{1}\right| \\
& >r_{x_{1} x_{2}}\left(1-\frac{\lambda}{2}\right) \geq \frac{r_{x_{1} x_{2}}}{2} .
\end{aligned}
$$

Thus

$$
\begin{aligned}
I_{3}\left(x_{1}, x_{2}\right) & \leq\left|x_{1}-x_{2}\right|^{2} \int_{\left\{\frac{r_{x_{1} x_{2}}}{2} \leq\left|x^{*}-y\right| \leq 2\right\}}\left|x^{*}-y\right|^{-4} d y \\
& \leq 4 \pi\left|x_{1}-x_{2}\right|^{2} \int_{\frac{r_{x_{1} x_{2}}}{2}}^{2} r^{-2} d r \\
& \leq 4 \pi\left|x_{1}-x_{2}\right| .
\end{aligned}
$$

Thus, we have proved

$$
\left\|L_{x_{1}}^{3}-L_{x_{2}}^{3}\right\|^{2} \leq \frac{C}{\pi}\left|x_{1}-x_{2}\right| .
$$

By computing $\nabla_{x} S^{3}$. $(y)$, we see that

$$
\left|\nabla_{x} S^{3}(y)\right|=4 \pi\left|S_{x}^{3}(y)\right|^{2}|y| \leq(4 \pi)^{-1}\left(1-\rho_{0}\right)^{-2}|y|, \forall x \in B_{\rho_{0}}(0) .
$$

Fix $x_{1}, x_{2} \in \bar{B}_{\rho_{0}}(0)$. The preceding inequality, along with the mean value theorem yields

$$
\begin{aligned}
\left\|S_{x_{1}}^{3}-S_{x_{2}}^{3}\right\|^{2} & =\int_{|y| \leq 1}\left(S_{x_{1}}^{3}(y)-S_{x_{2}}^{3}(y)\right)^{2} d y \\
& =\int_{|y| \leq 1}\left|\nabla_{x^{*}} S^{3}(y)\right|^{2}\left|x_{1}-x_{2}\right|^{2} d y \\
& \leq(12 \pi)^{-1}\left(1-\rho_{0}\right)^{-4}\left|x_{1}-x_{2}\right|^{2}
\end{aligned}
$$


where $x^{*}$ is a point lying on the interval determined by $x_{1}$ and $x_{2}$. Together with (62), this yields the upper bound in 59].

Let $\eta \in\left(0, \frac{1-\rho_{0}}{2 \rho_{0}} \wedge \frac{1}{2}\right)$. Since $\left|x_{1}-x_{2}\right| \leq 2 \rho_{0}$, we have $\eta\left|x_{1}-x_{2}\right|<1-\rho_{0}$. Let $D_{\eta}=\left\{y \in D:\left|y-x_{1}\right|<\eta\left|x_{1}-x_{2}\right|\right\}$. The choice of $\eta$ implies $D_{\eta} \subset D$, and then,

$$
\begin{aligned}
\left\|G_{D}^{3}\left(x_{1}, \cdot\right)-G_{D}^{3}\left(x_{2}, \cdot\right)\right\|^{2} & \geq\left\|G_{D}^{3}\left(x_{1}, \cdot\right)-G_{D}^{3}\left(x_{2}, \cdot\right)\right\|_{\eta}^{2} \\
& \geq \frac{1}{2}\left\|L_{x_{1}}^{3}-L_{x_{2}}^{3}\right\|_{\eta}^{2}-\left\|S_{x_{1}}^{3}-S_{x_{2}}^{3}\right\|_{\eta}^{2},
\end{aligned}
$$

where $\|\cdot\|_{\eta}$ denotes the $L^{2}$-norm on $D_{\eta}$.

With similar computations as in (64), we see that

$$
\begin{aligned}
\left\|S_{x_{1}}^{3}-S_{x_{2}}^{3}\right\|_{\eta}^{2} & =\int_{D_{\eta}}\left(S_{x_{1}}^{3}(y)-S_{x_{2}}^{3}(y)\right)^{2} d y \\
& =\int_{D_{\eta}}\left|\nabla_{x^{*}} S^{3}(y)\right|^{2}\left|x_{1}-x_{2}\right|^{2} d y \\
& \leq(4 \pi)^{-2}\left(1-\rho_{0}\right)^{-4}\left|x_{1}-x_{2}\right|^{2} \int_{D_{\eta}}|y|^{2} d y \\
& \leq(12 \pi)^{-1}\left(1-\rho_{0}\right)^{-4} \eta^{3}\left|x_{1}-x_{2}\right|^{5} .
\end{aligned}
$$

Next, we prove a lower estimate for $\left\|L_{x_{1}}^{3}-L_{x_{2}}^{3}\right\|_{\eta}^{2}$. Expanding the square of this norm yields,

$$
\left\|L_{x_{1}}^{3}-L_{x_{2}}^{3}\right\|_{\eta}^{2}=(4 \pi)^{-2}\left[J_{1}-2 J_{2}+J_{3}\right]
$$

with

$$
J_{1}=\int_{D_{\eta}} \frac{d y}{\left|y-x_{1}\right|^{2}}, J_{2}=\int_{D_{\eta}} \frac{d y}{\left|y-x_{1}\right|\left|y-x_{2}\right|}, J_{3}=\int_{D_{\eta}} \frac{d y}{\left|y-x_{2}\right|^{2}} .
$$

With a change of variables to spherical coordinates, we have

$$
J_{1}=4 \pi \int_{0}^{\eta\left|x_{1}-x_{2}\right|} d r=4 \pi \eta\left|x_{1}-x_{2}\right| .
$$

To study $J_{2}$, we notice that since $\eta<\frac{1}{2}$, we have $\left|y-x_{2}\right|>\frac{\left|x_{1}-x_{2}\right|}{2}$ for any $y \in D_{\eta}$. Indeed, assume that $\left|y-x_{2}\right| \leq \frac{\left|x_{1}-x_{2}\right|}{2}$, for some $y \in D_{\eta}$, then by the triangular inequality,

$$
\left|x_{1}-x_{2}\right| \leq\left|x_{1}-y\right|+\left|y-x_{2}\right| \leq\left(\eta+\frac{1}{2}\right)\left|x_{1}-x_{2}\right|,
$$

which is a contradiction. Hence, by applying spherical coordinates, we have

$$
\begin{aligned}
J_{2} & <\frac{2}{\left|x_{1}-x_{2}\right|} \int_{D_{\eta}} \frac{d y}{\left|y-x_{1}\right|}=\frac{8 \pi}{\left|x_{1}-x_{2}\right|} \int_{0}^{\eta\left|x_{1}-x_{2}\right|} r d r \\
& =4 \pi \eta^{2}\left|x_{1}-x_{2}\right| .
\end{aligned}
$$


Because $\eta<\frac{1}{2}$, we see that on the set $D_{\eta},\left|y-x_{2}\right|<(\eta+1)\left|x_{1}-x_{2}\right|<\frac{3}{2}\left|x_{1}-x_{2}\right|$. Thus,

$$
J_{3}>\frac{4}{9}\left|x_{1}-x_{2}\right|^{-2} \int_{D_{\eta}} d y=\frac{16 \pi}{27} \eta^{3}\left|x_{1}-x_{2}\right| .
$$

The estimates on the terms $J_{1}, J_{2}, J_{3}$ obtained above imply,

$$
\left\|L_{x_{1}}^{3}-L_{x_{2}}^{3}\right\|_{\eta}^{2}>(4 \pi)^{-1}\left|x_{1}-x_{2}\right| \eta\left(1-2 \eta+\frac{4}{27} \eta^{2}\right) .
$$

Along with [65), and since $\left|x_{1}-x_{2}\right|<2$, we obtain

$$
\begin{aligned}
& \left\|G_{D}^{3}\left(x_{1}, \cdot\right)-G_{D}^{3}\left(x_{2}, \cdot\right)\right\|^{2} \\
& \quad \geq(8 \pi)^{-1}\left|x_{1}-x_{2}\right|\left[\eta\left(1-2 \eta+\frac{4}{27} \eta^{2}\right)-\left|x_{1}-x_{2}\right|^{4} \eta^{3}\left(1-\rho_{0}\right)^{-4}\right] \\
& \quad \geq(8 \pi)^{-1}\left|x_{1}-x_{2}\right|\left[\eta\left(1-2 \eta-2^{4} \eta^{2}\left(1-\rho_{0}\right)^{-4}\right)\right],
\end{aligned}
$$

for any $\eta \in\left(0, \frac{1-\rho_{0}}{2 \rho_{0}} \wedge \frac{1}{2}\right)$.

Let $c_{1}=\frac{1-\rho_{0}}{2 \rho_{0}} \wedge \frac{1}{19} \wedge\left(1-\rho_{0}\right)^{4}$. The above computations show that, for any $\eta \in\left(c_{1} / 2, c_{1}\right)$,

$$
\left\|G_{D}^{3}\left(x_{1}, \cdot\right)-G_{D}^{3}\left(x_{2}, \cdot\right)\right\|^{2} \geq(8 \pi)^{-1} \frac{c_{1}}{38}\left|x_{1}-x_{2}\right| .
$$

This completes the proof of the lower bound in (59) and of the lemma.

Corollary 5.8 For $k=1,2,3$, we consider the setting of Lemmas 5.45 .55 .7 respectively. Then the mapping $x \mapsto \sigma_{x}=\left\|G_{D}^{k}(x, \cdot)\right\|$, is Hölder continuous. More precisely, there exists a constant $C>0$ such that, for any $x_{1}, x_{2} \in D$,

$$
\left|\sigma_{x_{1}}-\sigma_{x_{2}}\right| \leq C \begin{cases}\left|x_{1}-x_{2}\right|, & k=1 \\ \left|x_{1}-x_{2}\right|^{1-\gamma}, & k=2 \\ \left|x_{1}-x_{2}\right|^{\frac{1}{2}}, & k=3\end{cases}
$$

where $\gamma>0$ is arbitrarily small.

Proof: This is a consequence of the triangular inequality along with the upper bounds (43), 447), (59).

Remark 5.9 In connection with numerical approximations of the SPDE (6) with $d=1$ and $D=(0,1)^{k}, k=1,2,3$, we find in [12] the following results.

1. $\sup _{x \in D}\left\|G_{D}^{k}(x, \cdot)\right\|<+\infty([12$, Lemma 3.3 ] $)$. 
2. For any $\varepsilon>0$ there exists a constant $C=C(k, \varepsilon)$ (depending on $k$ and $\varepsilon$ ), such that, for any $x_{1}, x_{2} \in D$,

$$
\begin{aligned}
& \left\|G_{D}^{1}\left(x_{1}, \cdot\right)-G_{D}^{2}\left(x_{2}, \cdot\right)\right\| \leq C\left|x_{1}-x_{2}\right|^{1}, \\
& \left\|G_{D}^{2}\left(x_{1}, \cdot\right)-G_{D}^{2}\left(x_{2}, \cdot\right)\right\| \leq C\left|x_{1}-x_{2}\right|^{1-\varepsilon} \\
& \left\|G_{D}^{3}\left(x_{1}, \cdot\right)-G_{D}^{3}\left(x_{2}, \cdot\right)\right\| \leq C\left|x_{1}-x_{2}\right|^{\frac{1}{2}-\varepsilon} .
\end{aligned}
$$

(see [12, Lemma 3.4 ]).

The proof uses the development of the Green function with respect to an othonormal basis in $L^{2}(D)$.

\subsection{Hitting probabilities}

Throughout this section, we consider the following setting:

- Case $k=1 . D=(0, b), b>0, I$ is a closed interval of $D$ satisfying $d(I, \partial D)=b_{0}>0$.

- Case $k=2,3$. $D=B_{1}(0), I$ is a compact subset of $D$ satisfying $d(I, \partial D)=$ $d_{0}>0$.

\subsubsection{Upper bounds}

In this section, $A$ denotes a non empty Borel set of $\mathbb{R}^{d}$ and we establish upper bounds of the probability $\mathbb{P}\{v(I) \cap A \neq \emptyset\}$ in terms of the Hausdorff dimension of $A$.

Theorem 5.10 The sets $D \subset \mathbb{R}^{k}, I \subset D$ and $A$ are as above. Then, there exists a constant $C$, depending on $D, k, d$, such that

$$
\mathbb{P}\{v(I) \cap A \neq \emptyset\} \leq C \mathcal{H}_{d-\frac{k}{\xi}}(A),
$$

with

$$
\xi= \begin{cases}1, & k=1, \\ 1-\gamma, & k=2, \\ \frac{1}{2}, & k=3,\end{cases}
$$

where $\gamma>0$ is arbitrarily small.

Proof: If $d \leq \frac{k}{\xi}$, we have $\mathcal{H}_{d-\frac{k}{\xi}}(A)=\infty$, and (69) holds trivially.

Let $d>\frac{k}{\xi}$. We will apply [9, Theorem 2.6] to the process $\{v(x), x \in D\}$, which relies on the following assumptions:

(i) $\inf _{x \in K} \sigma_{x}>0$, for any compact subset $K \subset D$. 
(ii) For any $\epsilon$ small enough,

$$
\mathbb{E}\left(\int_{R_{j}^{\epsilon}} d x \int_{R_{j}^{\epsilon}} d y\left[\exp \left\{\frac{|v(x)-v(y)|}{|x-y|^{\xi}}\right\}\right]\right) \leq C \epsilon^{\frac{2 k}{\xi}},
$$

where $R_{j}^{\epsilon}=\Pi_{l=1}^{k}\left[j_{l} \epsilon^{\frac{1}{\xi}},\left(j_{l}+1\right) \epsilon^{\frac{1}{\xi}}\right), j=\left(j_{1}, \ldots, j_{k}\right), j_{1}, \ldots, j_{k} \in \mathbb{Z}$, and $R_{j}^{\epsilon} \cap I \neq \emptyset$.

Property (i) has already been proved. Hence, we put our efforts in proving (ii).

By the isometry property of the stochastic integral and Lemmas 5.4, 5.5, 5.7 (see the upper bounds in (43), (48), (59), respectively), we have

$$
\begin{aligned}
\mathbb{E}\left(|v(x)-v(y)|^{2}\right) & =\left\|G_{D}^{k}(x, \cdot)-G_{D}^{k}(y, \cdot)\right\|^{2} \\
& \leq C|x-y|^{2 \xi}
\end{aligned}
$$

with $\delta$ given in (70).

This implies

$$
\frac{\left|v^{i}(x)-v^{i}(y)\right|}{|x-y|^{\xi}} \leq C \frac{\left|v^{i}(x)-v^{i}(y)\right|}{\left[E\left(\left|v^{i}(x)-v^{i}(y)\right|^{2}\right)\right]^{\frac{1}{2}}},
$$

$i=1, \ldots, d$.

Let $\Lambda_{x, y}$ be the covariance matrix of the Gaussian random vector $v(x)-v(y)$, that is,

$$
\Lambda_{x, y}=\left(\left[E\left(\left|v^{i}(x)-v^{i}(y)\right|^{2}\right)\right]^{\frac{1}{2}} \delta_{i}^{j}\right)_{1 \leq i, j \leq d},
$$

$\delta_{i}^{j}$ being the Kronecker symbol.

The law of the random vector $Z:=\Lambda_{x, y}^{-1}[v(x)-v(y)]$ is $N_{d}(0$, Id $)$. Consequently,

$$
\begin{aligned}
\mathbb{E}\left(\int_{R_{j}^{\epsilon}} d x \int_{R_{j}^{\epsilon}} d y\left[\exp \left\{\frac{|v(x)-v(y)|}{|x-y|^{\xi}}\right\}\right]\right) & \leq C \int_{R_{j}^{\epsilon}} d x \int_{R_{j}^{\epsilon}} d y \mathbb{E}[\exp |Z|] \\
& \leq C \epsilon^{\frac{2 k}{\xi}}
\end{aligned}
$$

Hence, (71) holds.

\subsubsection{Lower bounds}

In this section, we consider the dimensions $k=1,3$. We refer to the introductory section for remarks relative to the dimension $k=2$. We have the following result.

Theorem 5.11 Let $k=1,3$. Fix $N>0$ and a Borel set $A \subset[-N, N]$. There exists a positive constant $c$ depending on the set $D$ and the parameters $d, N$, such that

$$
\mathbb{P}\{v(I) \cap A \neq \emptyset\} \geq c \operatorname{Cap}_{d-\frac{k}{\xi}}(A),
$$

with $\xi$ given in (70). 
Before giving the proof of this theorem, we observe that from (43), (59) and the definition of the pseudometric $\delta$ given in (40), we have

$$
c\left|x_{1}-x_{2}\right|^{\xi} \leq \delta\left(x_{1}, x_{2}\right) \leq C\left|x_{1}-x_{2}\right|^{\xi},
$$

for some positive constants $c, C$, and for any $x_{1}, x_{2} \in I$, where

$$
\xi= \begin{cases}1, & k=1 \\ \frac{1}{2}, & k=3 .\end{cases}
$$

Proof of Theorem 5.11] We apply [9, Theorem 2.1] to the stochastic process $v$ defined in (23). This accounts to check the following statements.

1. For any $x \in I$, the density function $z \mapsto p_{v(x)}(z)$ is continuous and bounded. Moreover, $p_{v(x)}(z)>0$ for any $z$ on a compact set of $\mathbb{R}^{d}$.

2. For any $x_{1}, x_{2} \in I, x_{1} \neq x_{2}$, the joint density of $\left(v\left(x_{1}\right), v\left(x_{2}\right)\right), p_{x_{1}, x_{2}}$, exists and satisfies this property:

Fix $M>0$. There exists $\gamma, \alpha>0$ such that $\frac{2}{\alpha}(\gamma-k)=d-\frac{k}{\xi}(\xi$ defined in (70), and

$$
p_{x_{1}, x_{2}}\left(z_{1}, z_{2}\right) \leq \frac{C}{\left|x_{1}-x_{2}\right|^{\gamma}} \exp \left(-\frac{c\left|z_{1}-z_{2}\right|^{2}}{\left|x_{1}-x_{2}\right|^{\alpha}}\right),
$$

for any $z_{1}, z_{2} \in[-M, M]^{d}$, where $C, c$ are positive constants independent of $x_{1}, x_{2}$.

Property 1 follows from (24)-27). Along with Lemma5.1, we infer the existence of the joint density $p_{x_{1}, x_{2}}$.

Case $k=1$

We fix $i \in\{1, \ldots, d\}$, and denote by $p_{x_{1}, x_{2}}^{i}\left(z_{1}, z_{2}\right), p_{x_{2} \mid x_{1}}^{i}\left(z_{2} \mid z_{1}\right), p_{x_{1}}^{i}\left(z_{1}\right)$ the joint density of $\left(v_{x_{1}}^{i}, v_{x_{2}}^{i}\right)$ at $\left(z_{1}, z_{2}\right)$, the conditional density of $v_{x_{2}}^{i}$ at point $z_{2}$ given $v_{x_{1}}^{i}=z_{1}$, and the marginal density of $v_{x_{1}}^{i}$ at $z_{1}$, respectively. Then, by linear regression,

$$
\begin{aligned}
p_{x_{1}, x_{2}}^{i}\left(z_{1}, z_{2}\right)= & p_{x_{2} \mid x_{1}}^{i}\left(z_{2} \mid z_{1}\right) p_{x_{1}}^{i}\left(z_{1}\right) \\
= & \frac{1}{\sqrt{2 \pi} \tau_{x_{1}, x_{2}}} \exp \left(-\frac{\left|z_{2}-m_{x_{1}, x_{2}} z_{1}\right|^{2}}{2 \tau_{x_{1}, x_{2}}^{2}}\right) \\
& \times \frac{1}{\sqrt{2 \pi} \sigma_{x_{1}}} \exp \left(-\frac{\left|z_{1}^{2}\right|}{2 \sigma_{x_{1}}^{2}}\right)
\end{aligned}
$$

where $m_{x_{1}, x_{2}}, \tau_{x_{1}, x_{2}}^{2}$ denote the conditional mean and variance, respectively (the definitions are recalled in Lemmas 5.2 and 5.3 . 
As in the proof of [9, Proposition 3.1], by simple algebraic manipulations, we obtain

$$
\begin{aligned}
p_{x_{1}, x_{2}}^{i}\left(z_{1}, z_{2}\right) \leq & \frac{1}{2 \pi \sigma_{x_{1}} \tau_{x_{1}, x_{2}}} \exp \left(-\frac{\left|z_{1}-z_{2}\right|^{2}}{4 \tau_{x_{1}, x_{2}}^{2}}\right) \\
& \times \exp \left(\frac{\left|z_{1}\right|^{2}\left|1-m_{x_{1}, x_{2}}\right|^{2}}{2 \tau_{x_{1}, x_{2}}^{2}}\right) \exp \left(-\frac{\left|z_{1}\right|^{2}}{2 \sigma_{x_{1}}^{2}}\right) .
\end{aligned}
$$

In order to get (75) (with $\frac{2}{\alpha}(\gamma-1)=d-1$ ) from (76), we will use (25), (38), and prove that

$$
c_{1}|x-y|^{2} \leq 1-\rho_{x y}^{2} \leq c_{2}|x-y|^{2},
$$

for any $x, y \in I$, where $c_{1}, c_{2}$ are positive constants.

The upper bound in (77) follows from (25) and (39), and is valid in any dimension $k$. A complete proof of (77) in dimension $k=1$ can be done as follows.

By definition,

$$
1-\rho_{x y}^{2}=\frac{\left(\sigma_{x} \sigma_{y}-\sigma_{x y}\right)\left(\sigma_{x} \sigma_{y}+\sigma_{x y}\right)}{\sigma_{x}^{2} \sigma_{y}^{2}} .
$$

Based on the expression (2), with direct computations we obtain

$$
\sigma_{x y}=\frac{x y}{6 b}\left(2 b^{2}-3 b x-3 b y+x^{2}+y^{2}\right)+\frac{x y(x \wedge y)}{2}-\frac{(x \wedge y)^{3}}{6},
$$

which yields

$$
\sigma_{x} \sigma_{y}-\sigma_{x y}=\frac{(x \wedge b)(b-(x \vee y))(x-y)^{2}}{6 b} .
$$

From the three equations above and 25], we deduce (77).

Going back to (76) and because of the independence of the components $v^{i}$, the estimates (77) imply the inequality (75) with $\gamma=d, \alpha=2$. This proves the lower bound (73) when $k=1$.

Case $k=3$

By Lemma 5.13, proved later on in this section, and (74), we obtain

$$
\left|\sigma_{x_{1}}^{2}-\sigma_{x_{2}}^{2}\right| \leq C \delta\left(x_{1}, x_{2}\right)^{1+\eta}
$$

with some $\eta>0$. This fact, together with (a) and (b) in Section 5.2 yields that the Gaussian stochastic process $\{v(x), x \in I\}$ satisfies the hypotheses of [9, Proposition 3.1]. Thus, according to that Proposition, if we fix $M>0$, for any $x_{1}, x_{2} \in I$, the joint density of $\left(v\left(x_{1}\right), v\left(x_{2}\right)\right)$ satisfies

$$
p_{x_{1}, x_{2}}\left(z_{1}, z_{2}\right) \leq \frac{C}{\left(\delta\left(x_{1}, x_{2}\right)\right)^{d}} \exp \left(-\frac{c\left|z_{1}-z_{2}\right|^{2}}{\left(\delta\left(x_{1}, x_{2}\right)\right)^{2}}\right)
$$


where $C, c$ are positive constants independent of $x_{1}, x_{2}$ and $z_{1}, z_{2} \in[-M, M]^{d}$. Because of (74), the right-hand side of the above inequality is bounded by

$$
\frac{C}{\left|x_{1}-x_{2}\right|^{\frac{d}{2}}} \exp \left(-\frac{c\left|z_{1}-z_{2}\right|^{2}}{\left|x_{1}-x_{2}\right|}\right) \text {. }
$$

Hence, Property 2. above holds with $\gamma:=\frac{d}{2}$ and $\alpha:=1$, which according to the conclusion of [9, Theorem 2.1] yields (73) for $k=3$.

Remark 5.12 Theorems 5.10 and 5.11 have the following consequences.

Let $k=1,3$. By the definition of the Hausdorff dimension $\operatorname{dim}_{\mathrm{H}}$ (see e.g. [16] or [14]) and Frostman's theorem, we have

$$
\begin{aligned}
& \operatorname{dim}_{\mathrm{H}}(A)<d-\frac{k}{\xi} \Longrightarrow\{v(I) \cap A=\emptyset\} \text { a.s. } \\
& \operatorname{dim}_{\mathrm{H}}(A)>d-\frac{k}{\xi} \Longrightarrow \mathbb{P}\{v(I) \cap A \neq \emptyset\}>0,
\end{aligned}
$$

with $\xi$ defined in (70).

Moreover, we see that if $d>\frac{k}{\xi}$, points $\left(A=\left\{y_{0}\right\}\right)$ are polar for $v$, while there are non polar if $d<\frac{k}{\xi}$.

If $d=\frac{k}{\xi}$ (critical dimension), the results obtained so far for the hitting probabilities are not informative. For example, if $A=\left\{y_{0}\right\}$, it says

$$
0 \leq \mathbb{P}\left\{\exists x \in I: v(I)=y_{0}\right\} \leq 1 .
$$

We refer to [5] for a method to characterise polarity of points for Gaussian random fields at critical dimensions with applications to the heat and wave stochastic equations.

In the particular case $k=2$, Theorem 5.10 implies that if $d>2$, points are polar for $v$. 5.11

We close this section with an auxiliary result used in the proof of Theorem

Lemma 5.13 Let $k=3$. Fix $\rho_{0} \in(0,1)$. There exists $C>0$ such that for all $x_{1}, x_{2} \in B_{\rho_{0}}(0)$,

$$
\left|\sigma_{x_{1}}^{2}-\sigma_{x_{2}}^{2}\right| \leq C\left|x_{1}-x_{2}\right|^{1-\zeta},
$$

with $\zeta>0$ arbitrarily small.

Proof: Let $r_{x_{1}, x_{2}}=2\left|x_{1}-x_{2}\right|$ and let

$$
\begin{aligned}
& D_{1}=D \cap\left\{\left|y-x_{1}\right| \leq r_{x_{1}, x_{2}}\right\}, \\
& D_{2}=D \cap\left\{\left|y-x_{1}\right|>r_{x_{1}, x_{2}}\right\} .
\end{aligned}
$$


By definition,

$$
\begin{aligned}
\sigma_{x_{1}}^{2}-\sigma_{x_{2}}^{2} & =\int_{D_{1}}\left[\left|G_{D}^{3}\left(x_{1}, y\right)\right|^{2}-\left|G_{D}^{3}\left(x_{2}, y\right)\right|^{2}\right] d y \\
& +\int_{D_{2}}\left[\left|G_{D}^{3}\left(x_{1}, y\right)\right|^{2}-\left|G_{D}^{3}\left(x_{2}, y\right)\right|^{2}\right] d y
\end{aligned}
$$

Consider the expression of $G_{D}^{3}$ given in (44). As observed in [13, p. 19], for all $x, y \in \bar{D}, G_{D}^{3}(x, y) \geq 0$ (notice that in the notation of that reference, $G_{D}^{3}(x, y)=$ $-G(x, y)$ ). Hence, with the notation (45), we have $S_{x}^{3}(y) \leq L_{x}^{3}(y)$, for any $x, y \in$ $\bar{D}$. Therefore,

$$
\begin{aligned}
& \left.\left|\int_{D_{1}}\left[\left|G_{D}^{3}\left(x_{1}, y\right)\right|^{2}-\left|G_{D}^{3}\left(x_{2}, y\right)\right|^{2}\right] d y\right| \leq 2 \int_{D_{1}}\left[\left(L_{x_{1}}^{3}(y)\right)^{2}+L_{x_{2}}^{3}(y)\right)^{2}\right] d y \\
& \quad \leq C\left(\int_{D_{1}}\left|x_{1}-y\right|^{-2} d y+\int_{D_{1}}\left|x_{2}-y\right|^{-2} d y\right) \\
& \quad \leq C\left|x_{1}-x_{2}\right|,
\end{aligned}
$$

where the last inequality follows from (60).

Our next aim is to find an upper bound for

$$
\left|\int_{D_{2}}\left[\left|G_{D}^{3}\left(x_{1}, y\right)\right|^{2}-\left|G_{D}^{3}\left(x_{2}, y\right)\right|^{2}\right] d y\right| .
$$

For this, we apply the mean value theorem to the function $x \mapsto\left(G_{D}^{3}(x, y)\right)^{2}$ and obtain

$$
\left(G_{D}^{3}\left(x_{1}, y\right)\right)^{2}-\left(G_{D}^{3}\left(x_{2}, y\right)\right)^{2}=2 G_{D}^{3}\left(x^{*}, y\right) \nabla_{x} G_{D}^{3}\left(x^{*}, y\right)\left(x_{1}-x_{2}\right),
$$

where $x^{*}=\lambda x_{1}+(1-\lambda) x_{2}$, for some $\lambda \in(0,1)$. This yields

$$
\begin{aligned}
& \left|\int_{D_{2}}\left[\left|G_{D}^{3}\left(x_{1}, y\right)\right|^{2}-\left|G_{D}^{3}\left(x_{2}, y\right)\right|^{2}\right] d y\right| \leq C\left|x_{1}-x_{2}\right| \\
& \quad \times \int_{D_{2}}\left|G_{D}^{3}\left(x^{*}, y\right)\right|\left|\nabla_{x} G_{D}^{3}\left(x^{*}, y\right)\right| d y .
\end{aligned}
$$

For all $x \in D$ and $\gamma \in(0,3)$, the integral $\int_{D}\left|G_{D}^{3}(x, y)\right|^{\gamma} d y$ is finite. Apply Hölder's inequality with $\gamma \in(0,3), \bar{\gamma}=\frac{\gamma}{\gamma-1}$ ( observe that $\bar{\gamma}>\frac{3}{2}$ ). We obtain

$$
\begin{aligned}
Z\left(x^{*}\right) & :=\int_{D_{2}}\left|G_{D}^{3}\left(x^{*}, y\right)\right|\left|\nabla_{x} G_{D}^{3}\left(x^{*}, y\right)\right| d y \\
& \leq\left(\int_{D_{2}}\left|G_{D}^{3}\left(x^{*}, y\right)\right|^{\gamma} d y\right)^{\frac{1}{\gamma}}\left(\int_{D_{2}}\left|\nabla_{x} G_{D}^{3}\left(x^{*}, y\right)\right|^{\bar{\gamma}} d y\right)^{\frac{1}{\gamma}} \\
& \leq C\left(\int_{D_{2}}\left|\nabla_{x} G_{D}^{3}\left(x^{*}, y\right)\right|^{\bar{\gamma}} d y\right)^{\frac{1}{\bar{\gamma}}} .
\end{aligned}
$$


We pursue the proof with the study of

$$
Y\left(x^{*}\right):=\int_{D_{2}}\left|\nabla_{x} G_{D}^{3}\left(x^{*}, y\right)\right|^{\bar{\gamma}} d y .
$$

Using the expression (46), we see that

$$
Y\left(x^{*}\right) \leq C\left(\int_{D_{2}}\left|\nabla_{x} L_{x^{*}}^{3}(y)\right|^{\bar{\gamma}} d y+\int_{D_{2}}\left|\nabla_{x} S_{x^{*}}^{3}(y)\right|^{\bar{\gamma}} d y\right) .
$$

Since

$$
\left|\nabla_{x} L_{x}^{3}(y)\right|=\left|\nabla_{x}\left(|x-y|^{-1}\right)\right|=|x-y|^{-2},
$$

and on the set $D_{2}$, we have $\left|x^{*}-y\right| \geq\left|x_{1}-x_{2}\right|$ (see (61), we obtain

$$
\begin{aligned}
\int_{D_{2}}\left|\nabla_{x} L_{x^{*}}^{3}(y)\right|^{\bar{\gamma}} d y & =\int_{D_{2}}\left|x^{*}-y\right|^{-2 \bar{\gamma}} d y \\
& \leq C \int_{\left|x_{1}-x_{2}\right|}^{3} r^{2-2 \bar{\gamma}} d r \\
& =\frac{C}{2 \bar{\gamma}-3}\left(\frac{1}{\left|x_{1}-x_{2}\right|^{2 \bar{\gamma}-3}}-\frac{1}{3^{2 \bar{\gamma}-3}}\right) \\
& \leq C\left|x_{1}-x_{2}\right|^{3-2 \bar{\gamma}}
\end{aligned}
$$

since $3-2 \bar{\gamma}<0$. By using (63), we have

$$
\int_{D_{2}}\left|\nabla_{x} S_{x^{*}}^{3}(y)\right|^{\bar{\gamma}} d y \leq C \int_{B_{2}(0)}|y|^{\bar{\gamma}} d y<\infty .
$$

Consequenly, we have proved that

$$
\begin{aligned}
\left|\int_{D_{2}}\left[\left|G_{D}^{3}\left(x_{1}, y\right)\right|^{2}-\left|G_{D}^{3}\left(x_{2}, y\right)\right|^{2}\right] d y\right| & \leq C\left(\left|x_{1}-x_{2}\right|+\left|x_{1}-x_{2}\right|^{1+\frac{3-2 \bar{\gamma}}{\bar{\gamma}}}\right) \\
& \leq C\left|x_{1}-x_{2}\right|^{1+\frac{3-2 \bar{\gamma}}{\bar{\gamma}}}
\end{aligned}
$$

because $3-2 \bar{\gamma}<0$.

The upper bound (82), along with (81) implies

$$
\left|\sigma_{x_{1}}^{2}-\sigma_{x_{2}}^{2}\right| \leq C\left|x_{1}-x_{2}\right|^{1+\frac{3-2 \bar{\gamma}}{\bar{\gamma}}} .
$$

By choosing $\gamma \in(0,3)$ arbitrarily close to 3 , we have $\bar{\gamma}>\frac{3}{2}$ and arbitrarily close to $\frac{3}{2}$. Thus, the exponent $1+\frac{3-2 \bar{\gamma}}{\bar{\gamma}}=\frac{3}{\bar{\gamma}}-1$ will be less than, but arbitrarily close to 1 . Hence, there exists $\eta>0$ such that (80) holds.

Acknowledgements. The authors express their gratitude to Joan Verdera, for useful guidance concerning the results of Section 5.3 . 


\section{References}

[1] H. Biermé, C. Lacaux, Y. Xiao. Hitting probabilities and the Hausdorff dimension of the inverse images of anisotropic Gaussian random fields. Bull. London Math. Soc. 1-21, (2009).

[2] V.I. Bogachev. Gaussian Measures. Mathematical Surveys and Monographs, Vol 62. American Mathematical Society, 1998.

[3] R. Buckdahn, E. Pardoux. Monotonicity methods for white noise driven quasilinear SPDEs, Diffusion processes and related problems in analysis, Vol.I: Diffusions in analysis and geometry, Proc. Int. Conf., Evanston/IL (USA) 1989, Prog. Probab. 22, 219-233 (1990).

[4] R.C. Dalang, E. Nualart. Potential for hyperbolic SPDEs. Ann. Probab. 32, 20099-2148 (2004).

[5] R.C. Dalang, C. Mueller, Y. Xiao. Polarity of points for Gaussian random fields. arXiv:1505.05417v1. Ann. Probab., to appear.

[6] R. C. Dalang, D. Khoshnevisan, E. Nualart. Hitting probabilities for systems of non-linear stochastic heat equations with additive noise. ALEA Lat. Am. J. Probab. Math. Stat. 3, 231-271 (2007).

[7] R. C. Dalang, D. Khoshnevisan, E. Nualart. Hitting probabilities for systems of nonlinear heat equations with multiplicative noise, Probability Theory and Related Fields, 144, 371-427 (2009).

[8] R. C. Dalang, M. Sanz-Solé. Hölder-Sobolev Regularity of the Solution to the Stochastic Wave Equation in Dimension Three, Memoirs of the American Mathematical Society, 199, no. 931 (2009).

[9] R. C. Dalang, M. Sanz-Solé. Criteria for hitting probabilities with applications to systems of stochastic wave equations, Bernoulli 16 (4) 1343-1368, (2009).

[10] R. C. Dalang, M. Sanz-Solé. Hitting probabilities for non-linear systems of stochastic waves. Memoirs of the American Mathematical Society, 237, Number 1120. September 2015.

[11] C. Donati-Martin. Quasi-linear Elliptic Stochastic Partial Differential Equation: Markov Property. Stochastics and Stochastics Reports, Vol. 41, pp. 219 240, (1992).

[12] I. Gyöngy, T. Martínez. On numerical solution of stochastic partial differential equations of elliptic type. Stochastics, 78(4), 213-231 (2006).

[13] D. Gilbarg, N.S. Trudinger. Elliptic Partial Differential Equations of Second Order. Springer, 2001. 
[14] D. Khoshnevisan. Multiparameter Processes: An Introduction to Random Fields. New York: Springer, 2002.

[15] S. Kusuoka. The non-linear transformation of a Gaussian measure on Banach space and its absolute continuity (I), J. Fac. Sci. Univ. Tokyo. Sec. IA, 567597, (1982).

[16] J.-P. Kahane: Some random series of functions. Cambridge University Press, 1993.

[17] S. Kwapień, J. Rosiński: Sample Hölder continuity of stochastic processes and majorizing measures. In: Seminar on Stochastic Analysis, Random Fields and Applications IV, pp. 155-163, Progr. Probab., 58, Birkhäuser, Basel 2004.

[18] M. Ledoux, M. Talagrand. Probability on Banach Spaces, Springer-Verlag, 1991.

[19] T. Martínez, M. Sanz-Solé. A Lattice Scheme for Stochastic Partial Differential Equations of Elliptic Type in Dimension $d \geq 4$. Appl. Math. Optim. 54:343-368, (2006).

[20] M. Reed, B. Simon. Functional analysis I. Methods of Modern Mathematical Physics, Academic Press, INC. (1980).

[21] M. Sanz-Solé, I. Torrecilla. A fractional Poisson equation: existence, regularity and approximations of the solution, Stochastic and Dynamics, Vol. 9, No. 4, 519-548, (2009).

[22] Y. Xiao. Sample path properties of anisotropic Gaussian random fields. In: A Minicourse on Stochastic Partial Differential Equations, (D. Khoshnevisan and F. Rassoul-Agha, editors), Lecture Notes in Math. 1962, 145212, Springer, New York, 2009.

[23] E. Zeidler. Nonlinear Functional Analysis and its Applications II/B. SpringerVerlag, 1990. 